\title{
Oggetti ordinari e straordinari. Nuovi documenti sulla produzione di argenti nella Napoli del secondo Seicento
}

\author{
Roberto Carmine Leardi \\ Università degli Studi di Salerno \\ robertoleardi@libero.it
}

Ricezione: 26/04/2019, Accettazione: 19/09/2019, Pubblicazione: 20/12/2019

\begin{abstract}
L'obiettivo di questo contributo è offrire una panoramica sulla produzione di oggetti d'argento nella Napoli del secondo cinquantennio del xvir secolo, attraverso nuovi documenti estratti dall'Archivio Storico del Banco di Napoli-Fondazione, appartenenti agli antichi banchi pubblici della capitale vicereale. Lo spoglio delle scritture contabili ha permesso di indagare le diverse tipologie, funzioni e destinazioni di questi prodotti, conosciuti unicamente in sede archivistica, e di evidenziare, dentro una cornice storico-sociale, il ruolo svolto dalla committenza. È emersa, pertanto, una netta prevalenza di manufatti religiosi, destinati all'arredo mobile e inamovibile di edifici di culto, rispetto alla suppellettile laica finalizzata alla magnificenza privata.
\end{abstract}

Parole chiave:

argenti; Napoli; Seicento; mecenatismo; Aniello Treglia; Gennaro Monte; Leonardo De Franco; Giovan Domenico Vinaccia

\section{ABSTRACT}

Ordinary and extraordinary objects: New documents on silverware production in late seventeenth-century Naples

The purpose of this paper is to offer an overview of silverware production in Naples during the second half of the seventeenth century through new documents found in the Historical Archive of the Banco di Napoli Foundation belonging to the old public banks of the viceregal capital. An examination of the accounting records has allowed us to investigate the different types, functions and destinations of these objects, known only in the archives and to highlight, within a historical and social framework, the role played by the commissioning client. The records reveal a clear prevalence of religious artifacts destined to the movable and unmovable furnishing of religious buildings compared to secular ornaments used for private luxury.

Keywords:

silverware; Naples; seventeenth century; patronage; Aniello Treglia; Gennaro Monte; Leonardo De Franco; Giovan Domenico Vinaccia 
$\mathrm{E}$ sigenze di rinnovamento, dispersioni e riutilizzo del materiale per finanziare campagne belliche, passando per le spoliazioni napoleoniche e i furti contemporanei, rendono la ricerca documentaria uno strumento essenziale per lo studio degli argenti ${ }^{1}$. Istrumenti notarili, testamenti, scritture contabili e, solo in parte, gli inventari, consentono di ricomporre il variegato scenario del secondo Seicento proteso tra sfera pubblica e privata, tra committenze laiche e religiose. Le opere sopravvissute rappresentano, com'è noto, una percentuale minoritaria di una produzione estremamente copiosa, benché difficilmente quantificabile soprattutto per l'ambito privato. L'intensa attività manifatturiera e il conseguente fenomeno delle esportazioni resero Napoli il massimo centro di approvvigionamento non soltanto per le province del Viceregno spagnolo ${ }^{2}$. Nella maggior parte dei casi questi oggetti, senza perdere la loro funzione pratica, diventavano un eccezionale strumento devozionale e di propaganda sociale, al pari di cicli ad affresco e pale d'altare. I principali destinatari furono gli edifici di culto che venivano omaggiati, con intenti ogni volta diversi, sia con argenti mobili, legati all'ufficio liturgico e a pratiche di culto, e sia quelli inamovibili, a integrazione di pitture e apparati plastico-architettonici. Oltre all'incomparabile magniloquenza della cappella del Tesoro di San Gennaro $^{3}$, un unicum al livello planetario, altri luoghi della città potevano vantare un patrimonio parimenti assortito, rimarcato senza sosta da cronisti contemporanei in preziosi volumi di periegesi.

\section{Statue di culto}

Nella produzione di argenti una posizione preminente era occupata dalle statue a mezzo busto, impiegate, solitamente, come contenitori di reli- quie ex-corpore di martiri, patroni/compatroni e nuovi santi della Controriforma, adattabili su pedagne d'argento, oppure di legno, per simulacri processionali, assecondando quel gusto per la teatralizzazione della religione peculiare dell'estetica barocca. Ciascuna chiesa, infatti, possedeva diversi esemplari che in occasioni solenni venivano sfoggiati sugli altari per pubblica ostensione, come le statue d'argento della basilica di Santa Chiara ricordate da Carlo De Lellis sull'altare maggiore ${ }^{4}$.

Tra le prime notizie emerse dallo spoglio documentario riportiamo quella concernente l'esecuzione di una testa, mani, diadema e giglio in argento di San Domenico di Guzmám, retribuiti il 3I agosto I665 dal frate predicatore Giovan Battista Guarino ad Aniello Treglia (notizie 1655-1672) dello scenario napoletano, attivo fin dal 1655 come autore di due «splendori d'argento» per Diego Chiros de Maiorca ${ }^{6}$. Circa tre anni dopo, il 6 luglio I668, l'argentiere otteneva da Serafino Spada ${ }^{7}$, membro della comunità di San Domenico Maggiore, la somma di io ducati, a fronte dei I 50 pattuiti, per una statua dello stesso santo, eletto nel I64 I patrono di Napoli, ma allo stato attuale non abbiamo dati sufficienti per collegarla al precedente manufatto ${ }^{8}$. Che in questo periodo Treglia godesse di proficui scampi con San Domenico Maggiore si evince dalla già nota statua di San Tommaso d'Aquino, saldata il 26 gennaio I $664^{9}$. L'opulenza delle chiese e dei conventi dell'Ordo fratrum praedicatorum viene sottolineata più volte dalle Notitie di Carlo Celano $^{10}$. Giungendo a San Domenico Maggiore, infatti, il canonico si mostra sbalordito dinanzi alla «quantità d'argenti lavorati in famose statue, come quella della Santissima Vergine del Rosario, tutta intera; quella di San Tomaso, 
dentro della quale si conserva la reliquia del suo braccio; oltre l'altra statua d'argento che sta nel nostro Sagro Tesoro, come nostro protettore; e quella di San Domenico» o al cospetto dei «famosi paleotti similmente d'argento, due gran torcieri, quantità di candelieri et altri vasi» ${ }^{11}$.

Ugualmente per una statua polimaterica in legno e argento dovrebbe riferirsi la polizza del I 8 gennaio I666 inoltrata da Carlo De Palma a Giovan Domenico Vinaccia (notizie I660I703), il nome più accreditato nel campo della statuaria d'argento della Napoli del secondo Seicento ${ }^{12}$. Formatosi a detta di Bernardo De Dominici nella scuola di Dionisio Lazzari, dove apprese il disegno delle figure e quello architettonico ${ }^{13}$, la sua versatilità lo condusse a spaziare, secondo un'ottica barocca, dall'architettura alla scultura in legno e bronzo, passando attraverso la progettazione di altari e cappelle ${ }^{14}$. Il documento in esame, che non riporta il materiale adoperato, riguarda l'esecuzione di una testa e mani della Vergine, oltre a un puttino, richiesti dall'anzidetto De Palma per la chiesa teatina di Santa Maria della Pietà a Ferrara ${ }^{15}$.

A distanza di un triennio, il certosino Francesco Maria Rocco, procuratore generale del monastero di San Lorenzo a Padula (Salerno), retribuiva Gennaro Monte (notizie I650-1697) per un San Bruno fatto «per devotione» dei monaci del cenobio, costato la cifra considerevole di I600 ducati ${ }^{16}$. Collaboratore in diversi cantieri di Cosimo Fanzago ${ }^{17}$, è assai probabile che il coinvolgimento in questa commessa extracittadina fosse dipeso dalla popolarità scaturita dalla partecipazione al cantiere dell'Annunziata di Napoli (1657) e, soprattutto, del Tesoro di San Gennaro (I66I I ${ }^{18}$. Questo «famosissimo scultore di metalli», celebrato da Giuseppe Artale con una poesia nell'Alloro fruttuoso del $1672^{19}$, allo scadere dello stesso anno veniva rimunerato da Luise Antonio Capasso per una Santa Giuliana di Nicomedia, pagata 250 ducati, da inviare nel casale di Frattamaggiore (Napoli) ${ }^{20}$, dove la martire vantava, assieme a San Sossio, del titolo di patrona.

L'ottavo e il nono decennio appaiono particolarmente dinamici per la produzione di busti d'argento, benché formulare letture generali risulti un'operazione complessa in assenza di studi sistematici.

In questa specializzazione una posizione rispettabile parrebbe stata ricoperta da Leonardo De Franco (notizie 1665-1675) 21, a cominciare dall'esecuzione nel r67I di un busto di San Biagio con pedagna per Maria Gabriella Pinto de Mendoza ${ }^{22}$, monaca domenicana dei Santi Pietro e Sebastiano di Napoli, del valore di 800 ducati ${ }^{23}$. A questa notizia nota aggiungiamo due polizze inedite. La prima, esattamente di un anno dopo, riferisce di un San Francesco Saverio, patrono di Napoli già dal I654, da collocarsi nella chiesa del Gesù Nuovo, saldato a De Franco dal gesuita Scipione Cacciuttolo per una spesa di i I 40 ducati ${ }^{24}$. La seconda è una commessa extracittadina del $\mathrm{r} 673$, anno in cui un tal padre Michele Caracciolo gli ordinava, per 530 ducati, un mezzo busto di San Giuseppe con pedagna di legno argentato per una «signora monaca» di Barletta ${ }^{25}$.

Parimenti indirizzato alla provincia era quel San Trifone martire, eseguito tra il ' 72 e il ' 73 dal mal noto Domenico De Gregorio per la chiesa parrocchiale di Santa Maria di Cesarano della terra di Tramonti (Salerno) ${ }^{26}$. Si trattava, credibilmente, di una statua votiva, richiesta in prima istanza ad Aniello Treglia da un tal Domenico De Vivo e lasciata incompleta a causa della morte dell'argentiere, per poi essere conclusa dal suddetto De Gregorio, in quanto suo probabile allievo/collaboratore, per interessamento dei discendenti Aniello e Onofrio De Vivo in ottemperanza alle disposizioni testamentarie del notabile, come si evince dalla cedola dell' I I agosto $1673^{27}$.

L'anno seguente, il i6 febbraio I674, Vincenzo d'Urso (notizie I674-1679) otteneva dal padre maestro Ignazio Genusio un saldo di 100 ducati, a fronte dei 200 pattuiti, per una scultura di Pio $\mathrm{V}^{28}$, beatificato due anni prima da papa Gregorio XIII, girando contestualmente la medesima somma a un certo Nicola Maramaldo, in conto «dell'argento, et manifattura» di una statua del beato Ghislieri con camauro d'argento "senza però testa, et mani» ${ }^{29}$, da riconoscersi in quella osservata dalla guidistica nella sagrestia di San Domenico Maggiore ${ }^{30}$. Quest'ultima operazione sembrerebbe un vero e proprio subappalto, dove d'Urso si sarebbe accollato l'esecuzione delle porzioni fondamentali - testa e mani -, lasciando al collega quelle meno impegnative per contenere le spese o velocizzare i tempi di consegna. Un biennio dopo lo stesso argentiere sarà ricompensato dal predicatore Giuseppe Conte per un San Domenico, costato 250 ducati $^{31}$, ma di queste statue non conosciamo le destinazioni. È lecito immaginare che possa trattarsi della comunità di San Domenico Maggiore, con la quale d'Urso ebbe una controversia penale fino al punto da essere incarcerato per il mancato rispetto delle condizioni contrattuali relative a un paliotto d'argento, commissionatogli prima del i680 dallo stesso Genusio ${ }^{32}$.

Tra le polizze del nono decennio risulta significativo un mezzo busto di San Celestino V, pagato nel I680 da una principessa di Santobuono, forse Giovanna Caracciolo consorte di Marino V. Il documento, a parte non precisare 


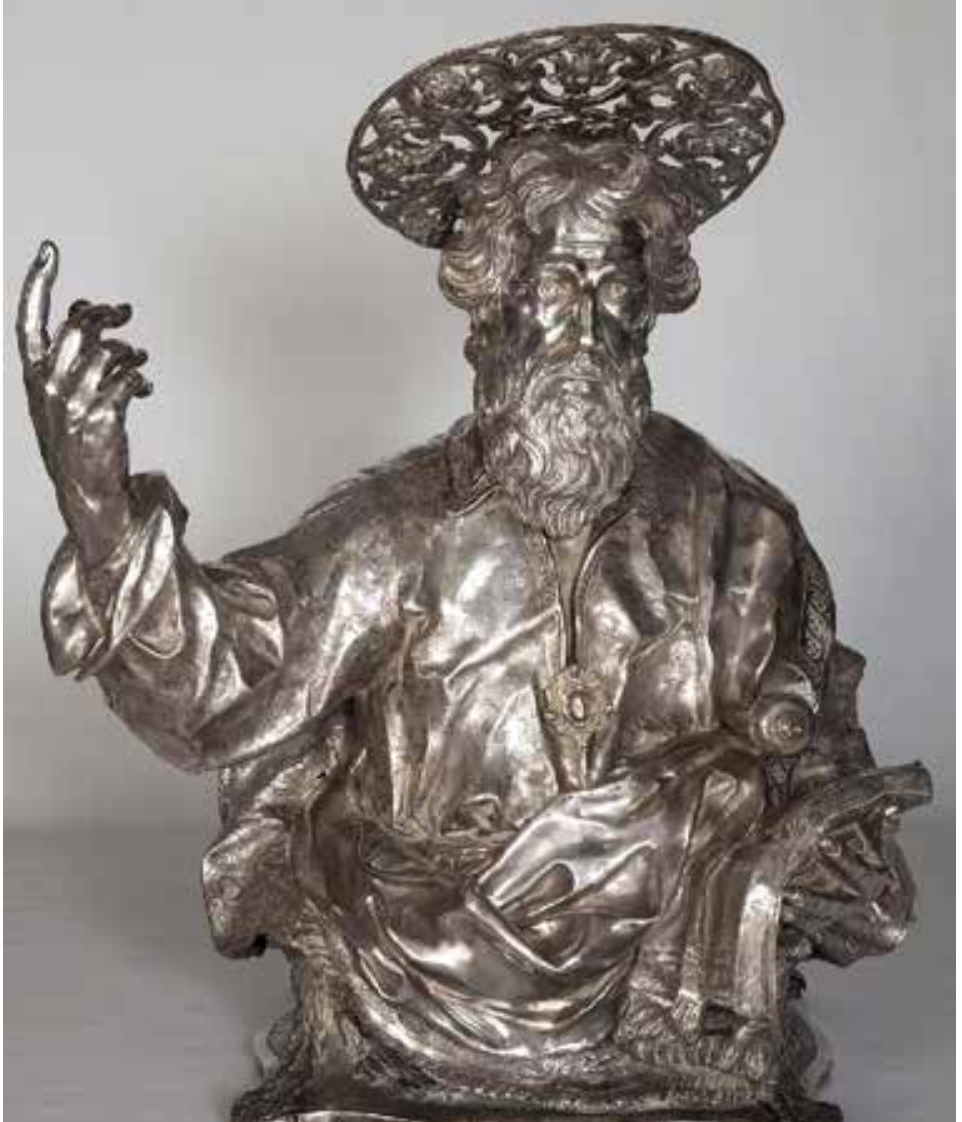

Figura 1.

Giovan Domenico Vinaccia, Busto di San Paolo Apostolo. Aversa (Caserta), Cattedrale di San Paolo Apostolo.

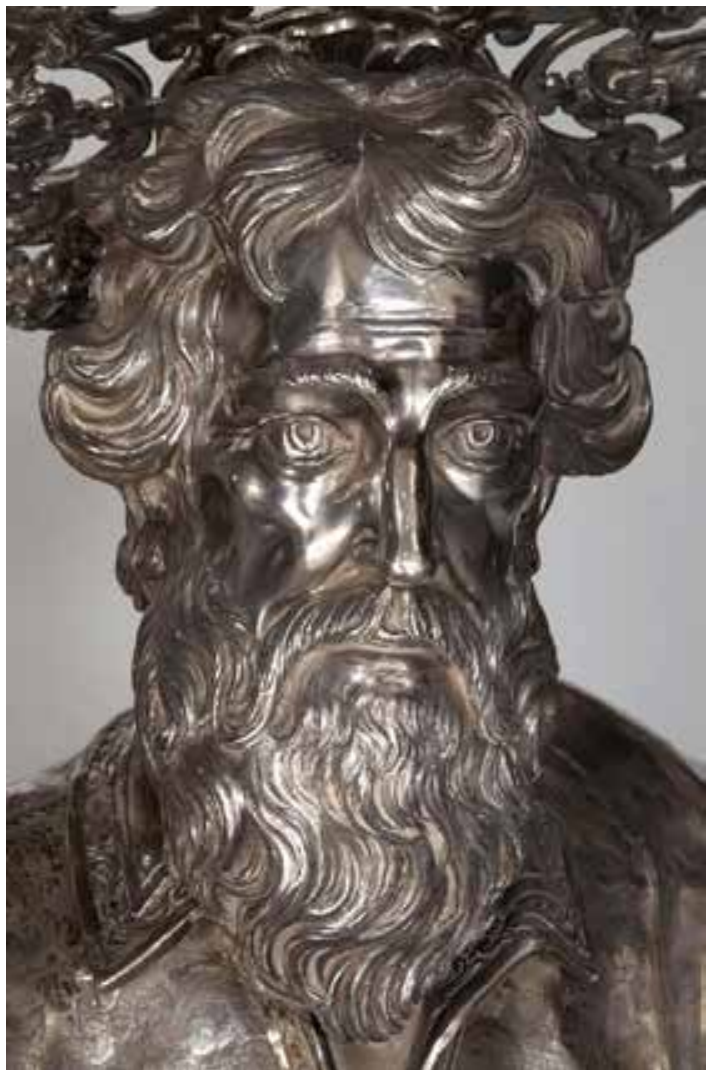

Figura 2.

Giovan Domenico Vinaccia, Busto di San Paolo Apostolo, particolare. Aversa (Caserta), Cattedrale di San Paolo Apostolo. l'ubicazione, credibilmente la chiesa partenopea di San Pietro a Maiella ${ }^{33}$, omette perfino il nome dell'argentiere, riportando esclusivamente quelli dei monaci celestini Francesco Borgia e Domenico d'Agostino ${ }^{34}$.

Per l'anno i68 I sono emerse quattro cedole di pagamento. Il 29 gennaio Beatrice Ventimiglia, moglie di Leonardo VI principe d'Acaia, retribuiva in qualità di «madre, balia e tutrice» del primogenito Carlo Antonio Tocco, futuro principe d'Acaia e Montemiletto, lo scultore Vinaccia per una statua di Sant'Anna, protettrice della famiglia, associabile a quella che si conservava nella cappella del loro palazzo contenente la reliquiae ex-notabilis del piede della sant ${ }^{35}$. La scultura, come si legge nel documento, doveva soddisfare esigenze devozionali, essendo un ex-voto del giovane rampollo alla madre della Vergine per recuperare la salute fisica ${ }^{36}$.

Anche se spesso non disponiamo d'informazioni specifiche, molti manufatti venivano compiuti recuperando pezzi più antichi guastati dal tempo o ritenuti demodé. È il caso della Santa Fortunata con pedagna, pagata il io febbraio I68 I con un acconto di ioo ducati a Gennaro Monte da una certa Margherita de' Rossa, riutilizzando l'argento di una vecchia scultura della martire in suo possesso, munita degli attributi del libro, palma e corona ${ }^{37}$.

Fra i maestri del secondo Seicento è indubbiamente Vinaccia a detenere la leadership nella «scultura e 'l gettar de' metalli», come notava anche De Dominici ${ }^{38}$. Dalla documentazione sinora raccolta, costui figura come il più prolifico esecutore di statue e busti d'argento, condividendo il palcoscenico con De Franco, Gennaro Monte e Aniello Treglia. Tra gli anni Settanta e Ottanta, benché sia arduo riassumere la messe archivistica, notiamo una netta prevalenza di commesse provenienti dalle sedi napoletane della Societas Iesu, come le statue di San Francesco Borgia (I67I-72 $)^{39}$ e Sant'Ignazio di Loyola $(\mathrm{I} 678-79)^{40}$. Tra le nuove tracce segnaliamo quella di 200 ducati, risalente al 9 giugno I68 I, relativa a un busto di San Paolo Apostolo, commissionato da Paolo Carafa della Spina, vescovo di Aversa dal I665 al I68641. Tale ritrovamento, assieme a un'altra recente segnalazione ${ }^{42}$, consente di riconoscere il manufatto in quel tutt'oggi venerato nella cattedrale della città casertana (figura $\mathrm{I}-2)^{43}$, linguisticamente affine, per gusto e attitudine, ai busti di San Giovanni Battista e Santa Maria Egiziaca del Tesoro di San Gennaro, modellati allo scadere del Seicento da Lorenzo Vaccaro $^{44}$. Sempre nel I68 I sono affiorati diversi pagamenti a Vinaccia provenienti dall'Oratorio partenopeo per una generica statua $a^{45}$, forse un'effige aggiornata del fondatore San Filippo Neri da collocarsi ai Girolamini, nonostante 
fosse già stata compiuta da Onofrio d'Alessio una per il complesso filippino e una seconda da Aniello Treglia per il pantheon dei protettori della città dentro il Tesoro gennareo ${ }^{46}$.

\section{Arredi chiesastici e di rappresentanza}

All'interno degli edifici sacri i principali investimenti finanziari si focalizzavano sull'arredo mobile e inamovibile degli altari, in modo particolare su quello maggiore, soggetto più di tutti a trasformazioni per esigenze liturgiche e di consolidamento dei culti, oltre alla sistematica azione propagandistica derivante dall'avvicendamento di religiosi alle diverse posizioni gerarchiche. Lo sfarzo dei luoghi di culto della capitale non poteva lasciare indifferenti gli autori delle numerose guide cittadine. Tra $\mathrm{i}$ casi più significativi, ad esempio, l'abate Pompeo Sarnelli e Celano annoveravano la sagrestia dei Girolamini, per «la ricchezza degl'argenti per ornamento degl'altari, consistente in candelieri e statue che fra questi si frapongono, vasi con li loro fiori, e fra questi vi si veggono quattro torcieri d'argento che da noi si chiamano splendori» ${ }^{47}$, e quella dell'Annunziata, per il «maraviglioso guardarobba degl'argenti, che al certo simile non se ne vede in Italia ${ }^{48}$.

A tal proposito risulta emblematico un documento del 9 ottobre I 687 sorprendente per la descrizione minuziosa degli oggetti, concernenti in un servizio di candelieri, alti palmi $4 \mathrm{e}$ del peso di ro libbre circa, commissionati a Nicola d'Aula da suor Maria Rosalea Mercurio, membro plausibilmente del monastero della Concezione a Montecalvario. Tra le condizioni imposte all'argentiere si legge l'obbligo di prendere a modello il nuovo corredo di candelieri di San Pietro a Maiella, oltre a ciò, su ciascuna pedagna avrebbe dovuto effigiare da una parte l'Immacolata Concezione, con la sua «coronella in testa indorata», e lo stemma della famiglia Mercurio, e dall'altra il nome della monaca blasonata ${ }^{49}$.

Altro oggetto riservato agli altari, qualora non fosse previsto un tronetto per l'adorazione dell'Eucarestia, era la grande croce posta alla sommità a ricordo del sacrificio salvifico di Cristo. Un esemplare del suddetto d'Aula, con sotto il monte Calvario, svettava sull'altare maestro della chiesa di Sant'Andrea delle Dame di Napoli, richiesto allo scadere del ' 73 da Chiara Maria di Bologna, priora del monastero ${ }^{50}$.

Un manufatto simile di Vinaccia, anche se non possiamo escludere una funzione processionale, veniva spedito nel 1682 alla chiesa di Santa Maria del Monte a Teggiano (Salerno) ${ }^{51}$, per interessamento dell'abate Gennaro Filo- marino $^{52}$, rettore della cappella di Santa Maria dei Vulcani di Napoli53. L'anno seguente costui rimunererà ancora Vinaccia per un parato di sei candelieri realizzati per la chiesa napoletana della Santissima Trinità delle Monache ${ }^{54}$.

Anche le custodie eucaristiche, in piena adesione ai dettami conciliari e al capitolo xiII delle Instructiones fabricae et supellectilis ecclesiasticae di Carlo Borromeo, del $1577^{55}$, diventavano sfoggio di ricchezza, soprattutto le facciate anteriori con le proprie portelle ${ }^{56}$. Singolare era quella della chiesa della certosa di San Martino, «tutto a gitto» di Vinaccia, costata la strabiliante cifra di 6000 scudi e recensita da gran parte della guidistica $^{57}$.

Tra i nuovi esemplari abbiamo notizia di uno con puttini, sempre di Vinaccia, per la chiesa dell'eremo dei Camaldoli di Nola (Napoli) ${ }^{58}$, saldato il 30 gennaio i 675 assieme a un corredo di giare e candelieri di legno - ingessati, dipinti e parzialmente argentati ${ }^{-59}$, o quello spedito a Bari da De Franco per l'altare di San Nicola, forse nell'omonima basilica del santo, testimoniato dalla polizza del 27 marzo $1675^{60}$.

Per contenere le spese in molti casi l'argento cedeva il posto al più economico, e meno nobile, rame indorato, come nel bassorilievo dello scultore Domenico Marinelli (notizie I662I687) $)^{61}$, incorporato nella custodia della chiesa dell'Ave Gratia Plena di Gaeta (Latina), alla cui realizzazione sovrintese Dionisio Lazzari ${ }^{62}$.

Persino alcuni tronetti per l'adorazione del Sacramento presentavano una manifattura in argento, come quello corrisposto nel $\mathrm{I}_{674}$ a Vinaccia per la chiesa del Gesù Nuovo ${ }^{63}$, plausibilmente per l'altare maggiore, o quello commissionato nel I68 I allo scultore Andrea Finelli da Vittoria e Maria Coppola per un edificio imprecisato ${ }^{64}$.

Dotato all'incirca della stessa funzione era quel baldacchino, in rame indorato e argento, ideato nel ' 72 da Monte per la chiesa napoletana di Santa Maria della Sapienza su iniziativa disuor Maria Fortunata Caracciolo ${ }^{65}$, dei duchidi Sicignano ${ }^{66}$, dove invece Celano segnalava una «custodia d'argento massiccio, disegno et opera» di Vinaccia ${ }^{67}$.

Ingenti risorse venivano altresì impiegate nell'abbellimento di altari dedicati a santi o icone prodigiose intronizzate, secondo logiche propagandistiche e di rilancio dei culti. È il caso della considerevole somma investita da Violante di Sangro dei principi di Sansevero, consorella del monastero di San Gaudioso, che allo scadere del I 665 retribuiva De Franco per una coppia di sgabelli con due statue d'argento, «et gradina o piedistalli», per l'altare di Santa Maria d'Agnone, analogamente d'argento, sito nell'anzidetto cenobio partenopeo ${ }^{68}$. 


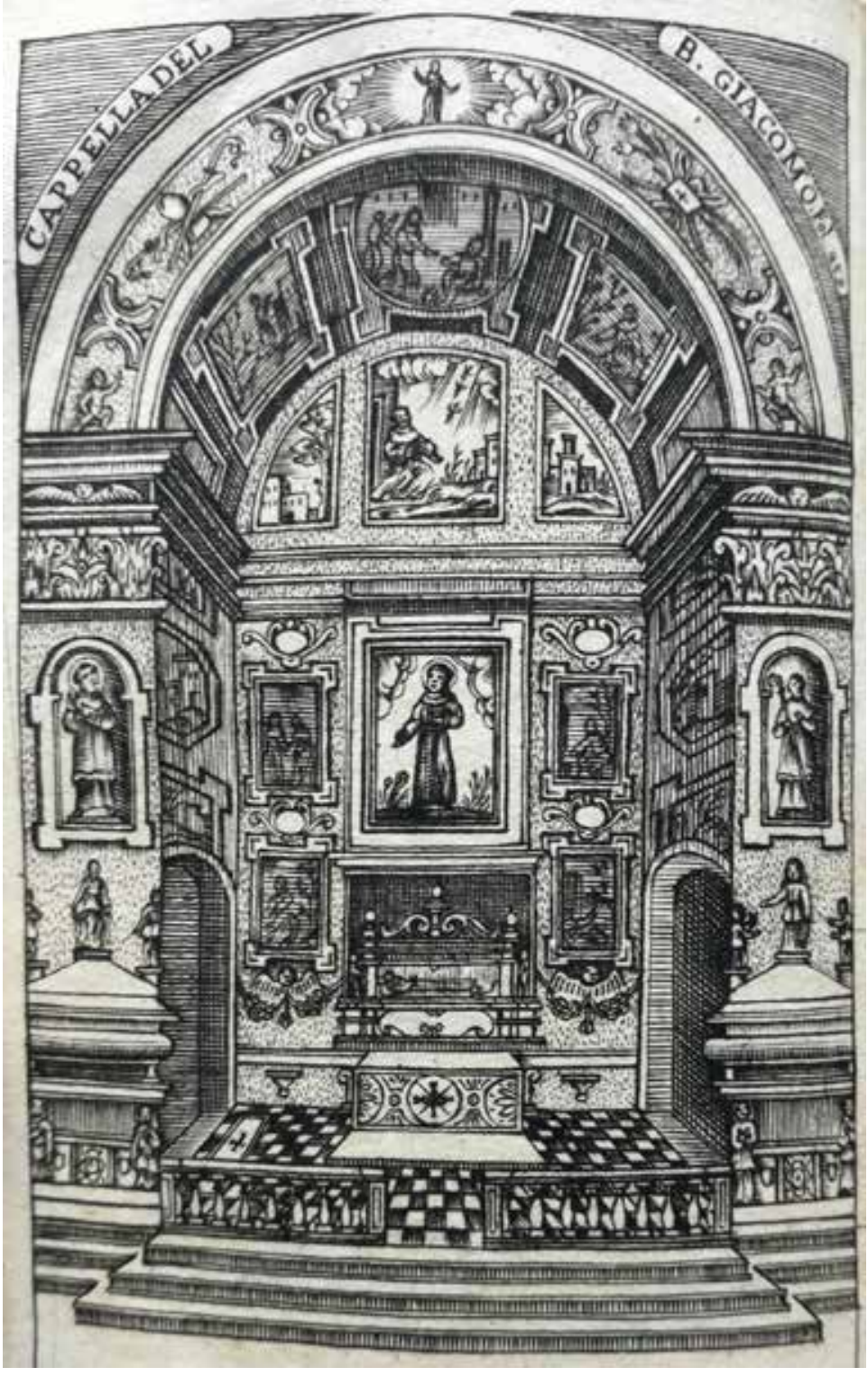

Figura 3.

Cappellone di San Giacomo della Marca. Da P. SARnelli, Guida de' forestieri curiosi di vedere e d'intendere le cose più notabili della regal città di Napoli e del suo amenissimo distretto, Napoli, Rosselli, 1685. (foto: Biblioteca di Nazionale di Napoli)

Un altro luogo fondamentale della spiritualità partenopea era il cappellone di San Giacomo della Marca, patrono di Napoli fin dal I647, annesso alla chiesa di Santa Maria la Nova, una delle principali sedi francescane osservanti della capitale (figura 3). Tra il '69 e il '7r si procedeva all'abbellimento dell'edificio con due angeli argentei di Gennaro Monte, forse da collocare ai lati dell'arca contenente il corpo «intero et incorrotto» del santo $^{69}$, sovvenzionati con le elemosine raccolte dal sacrestano Paolo da Montesarchio ${ }^{70}$.

Sempre dentro Santa Maria la Nova, un discorso analogo vale per l'icona prodigiosa della Madonna delle Grazie, dalla quale si ricevevano "gratie infinite, e di continuo vi vengono grandi elemosine, delle quali una gran parte se ne spende agl'ornamenti della chiesa, come si vede, e fin hora vi sono da 30 mila scudi di peso d'argento ${ }^{71}$. Per il «nobile baldacchino d'argento massiccio» della cappella (figura 4$)^{72}$, Nicola d'Aula fabbricò nel i67I una cornice, assieme ad alcuni candelieri da posizionarsi probabilmente sull'altare ${ }^{73}$, sotto il quale verrà successivamente posizionato il pregevole paliotto iniziato da Marinelli e concluso da Matteo Treglia ${ }^{74}$.

Un'attenzione circostanziata richiede quel baldacchino in argento e rame indorato, oggi non più esistente, della Madonna Bruna, effige «bella e divota» della basilica del Carmine Maggiore (figura 5$)^{75}$, affidato nel 1675 all'argentiere Antonio Monaco (notizie I670-I674) da Carlo Grimaldi dei principi di Gerace. Tale lavoro sarà in seguito periziato da Tommaso Cavallo e, nientemeno, da Vinaccia per interessamento di Filippo Grimaldi, il quale ottenne in omaggio dal consanguineo una piccola croce e uno specchio d'argento dello stesso maestro ${ }^{76}$.

Una posizione leader nella produzione di argenti era occupata dall'oggettistica ordinaria per cerimonie liturgiche, che spesso accompagnava, come un vero e proprio corredo personale, gli spostamenti degli ecclesiastici. Lo scavo archivistico ha consegnato un'interessante polizza, datata 3 aprile 1665 , concernente l'acquisto da parte di Carlo De Angelis, vescovo dell'Aquila dal i663 al i674, di un baculo pastorale e due baldacchini, di palmi io e I2, guarniti rispettivamente di velluto cremosino napoletano e d'armesi cremosino, tutti dell'orefice Andrea Nauclerio ${ }^{77}$. Invece, monsignor Ottavio Paravicino, titolare dal I68 I al i696 della diocesi calabrese di Mileto, ordinava a Domenico Antonio Fera, tramite il congiunto Giovanni Matteo, due profumiere e una mezza corona ${ }^{78}$.

Tra i manufatti legati a una funzione di rappresentanza abbiamo traccia il 23 giugno I67I di una sfera d'argento, con sopra una croce, ideata da Domenico Gigante per il gonfalone dell'ospizio dei Santi Pietro e Gennaro ${ }^{79}$.

\section{Panni d'altare}

Tra i complementi più utilizzati vi era il paliotto, lavorato perlopiù a sbalzo e a fusione che, solitamente, trovava sistemazione in quei luoghi della chiesa oggetto di una sentita pietas popolare - l'altare maggiore o di cappelle dedicate a un'icona/reliquia prodigiosa - o per solenni festeggiamenti ${ }^{80}$. Applicato sul fronte anteriore, al di sotto della mensa, l'antependium esibiva comunemente un corredo iconografico, inciso e a rilievo, capace di assecondare logiche propagandistiche di promozione di un culto o una congregazione religiosa. L'esame delle scritture apodissarie ha riportato alla luce numerose atte- 


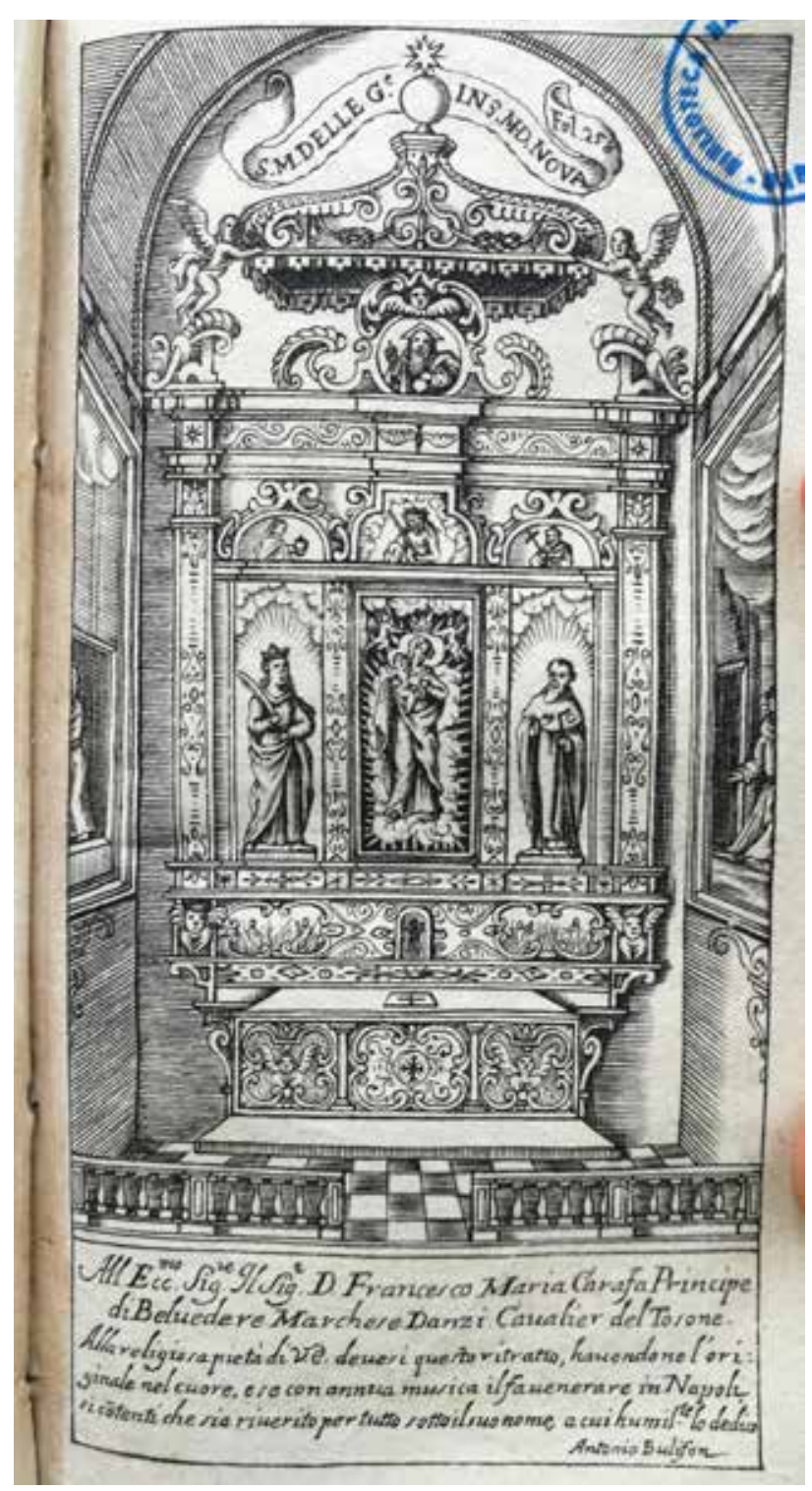

Figura 4.

Cappella di Santa Maria delle Grazie. Da P. SARnelli, Guida de' forestieri curiosi di vedere e d'intendere le cose più notabili della regal città di Napoli $e$ del suo amenissimo distretto, Napoli, Rosselli, 1685. (foto: Biblioteca di Nazionale di Napoli)

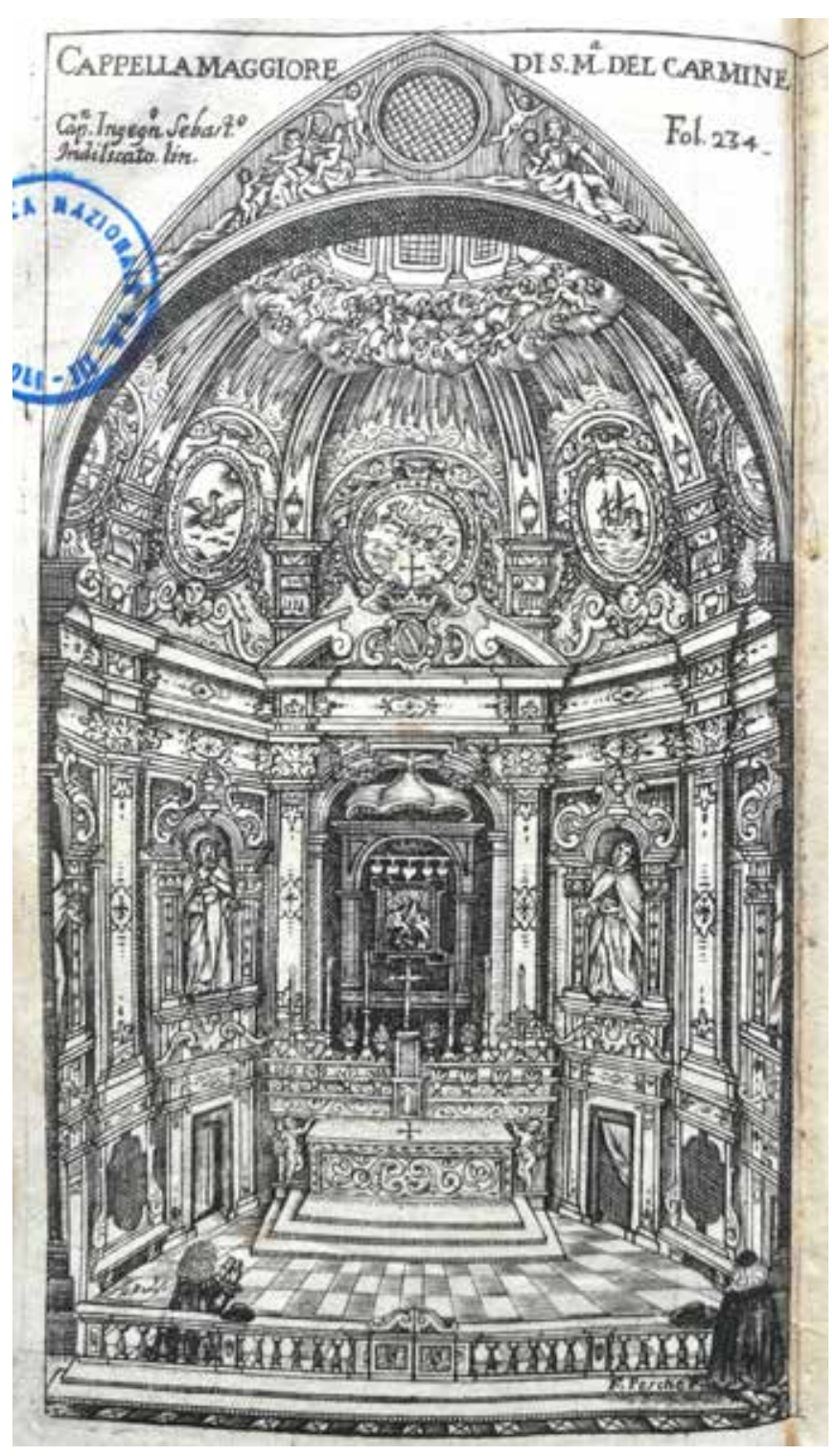

Figura 5.

Altare maggiore della chiesa di Santa Maria del Carmine Maggiore. Da P. SARNELLI, Guida de' forestieri curiosi di vedere e d'intendere le cose più notabili della regal città di Napoli e del suo amenissimo distretto, Napoli, Rosselli, 1685. (foto: Biblioteca di Nazionale di Napoli) stazioni inedite che, sommate a quelle già edite, contribuiscono a delineare un panorama assai variegato per il secondo Seicento.

La prima traccia ci conduce alla chiesa di Santa Maria di Piedigrotta, uno dei più antichi santuari mariani del Vicereame di fondazione angioina, sorto attorno alla statua lignea della Madonna Theotókos Odigitria e amministrato dai canonici regolari lateranensi del Santissimo Salvatore. Uno di questi, un tal Innocenzo de Forte, elargiva un ultimo pagamento al mal noto Francesco Califano, riscosso il i6 settembre I666, per il paliotto dell'altare maggiore costato la ragguardevole cifra di circa 626 ducati ${ }^{81}$.

Che le diverse botteghe della città costituirono un essenziale punto di riferimento per l'intero Viceregno è cosa ben nota alla storiografia, supportata da numerose emergenze archivistiche difficilmente riassumibili. A queste aggiungiamo la notizia di un paliotto «traforato e corniciato» del peso di i 8 libbre, lungo 9 e largo 4 palmi, approntato da Vinaccia per l'altare del Santissimo Sacramento della chiesa parrocchiale di Modugno (Bari), come si evince dalla polizza di Giovanni Pietro Manara del I4 luglio $\mathrm{I} 672^{82}$.

Ancora per la provincia è quel panno d'altare, costato 600 ducati, ordinato a Giuseppe d'Aula dal principe Carlo de Mari, ma la cedola del 2 I giugno I673 omette l'ubicazione ${ }^{83}$, probabilmente qualche edificio ecclesiastico dei suoi feudi pugliesi ${ }^{84}$. 
A distanza di pochi giorni, Leonardo De Franco ritirava un saldo di 200 ducati, a fronte dei 200 stimati, per un paliotto e corredo di sei candelieri voluti da Mauro Cesarini di Nola, abate dal I 669 al i675 dell'archicenobio benedettino di Montecassino ${ }^{85}$. Dall'istrumento notarile sappiano che il primo manufatto, da disporsi innanzi all'altare del fondatore della Regula monachorum, doveva essere di palmi 4 d'altezza e 8 di larghezza, in argento «di piastra a martello» con «tutte le teste di cherubini di getto»e tre figure al centro di «mezzo relievo» ${ }^{86}$.

Per il secondo cinquantennio, come già visto a proposito delle statue di culto, appare alquanto movimentata la domanda da parte dell'ordine medicante di San Domenico, impegnati ad abbellire con grande lusso i principali edifici della città, diversamente della maggiore sobrietà perseguita dalla famiglia serafica di San Francesco d'Assisi. È, infatti, il caso del paliotto della chiesa di San Pietro martire, eseguito per l'altare maggiore credibilmente in stoffa ricamata, ma che nel 1682 accolse una figura d'argento del martire veronese compiuta da Matteo Treglia ${ }^{87}$, figlio di Aniello, come indicato nella polizza di fra Nicola di Maddalonis8. Nello stesso anno Domenico Maria Marchesi, frate di San Domenico Maggiore, assoldava Vinaccia, giunto oramai all'apice della sua carriera, per la fattura di un paliotto di 400 ducati da porsi sull'altare maggiore, come si evince dalla cedola del 5 settembre $1682^{89}$. Invero, già l'anno precedente, lo retribuiva per una custodia d'argento da destinarsi alla chiesa napoletana di San Tommaso d'Aquino ${ }^{90}$, segnalata anche da Sarnelli ${ }^{91}$, e, sempre nello stesso luogo, per un paliotto d'argento $^{92}$ e una statua lignea dell'Angelicum con inserti in rame indorato e «testa, mani, giglio, sfera, diadema e reliquiario» d'argento ${ }^{93}$.

\section{Argenti privati}

La produzione di argenti indirizzata ai privati rappresenta il segmento più interessante per l'assortimento dei manufatti, sebbene più complesso da ricostruire a causa della natura approssimativa degli strumenti di ricerca, in primis gli inventari. Al loro interno, infatti, l'oggettistica artistica spesso si mescolava indistintamente agli utensili di uso quotidiano, oppure rubricata in modo generico sotto la voce argenteria o galanteria. Descrizioni più dettagliate, benché variabili da caso a caso, possono essere estrapolate dalle scritture apodissarie. Queste hanno consegnato una varietà di suppellettili e fantasiose creazioni in grado di assecondare esigenze devozionali, come immagini sacre e acquasantiere, ma anche l'attitudine all'arre- do frivolo e capriccioso di certi privati, non soltanto d'estrazione aristocratica. Se Giovan Battista Amendola, consigliere presidente della Regia Camera della Sommaria, acquistava nel ' 57 per circa I 40 ducati due Villani e un Orfeo con «diversi animalucci d'argento» da Giuseppe d'Andrea ${ }^{94}$, nel decennio successivo il principe di Cassano richiedeva una fontana d'argento a Gennaro Monte, con un acconto di 50 ducati del io ottobre $1665^{95}$.

Alla sfera privata parrebbe rivolgersi prevalentemente Domenico Antonio Fera (notizie I665-1682), consanguineo credibilmente dell'argentiere Bernardino, attivo a Napoli tra il I640 e il I $650^{96}$. All'attività precoce dovrebbe risalire quel Presepe d'argento, del peso di 177 libbre, pagato nel i665 dal marchese d'Altavil$\mathrm{la}^{97}$, quasi sicuramente Pompeo Romano, e le due statue per Carlo Mormile, costate circa 2 Is ducati98. Alla tarda produzione, anche se non disponiamo di una precisa scansione dei suoi lavori, potrebbe ricondursi la coppia di carri trionfali raffiguranti il mare e la terra, di circa 20 libbre, consegnate al duca di Diano nelle loro "cassette», che il I6 maggio I68 I gli fruttarono la somma di ioo ducati per la sola manifattura ${ }^{99}$. In questo frangente, a conferma della sua inclinazione per il mercato privato, realizzava per Ignazio Provenzale, duca di Collecorvino ${ }^{100}$, un «caprio e una cerva» e un Atlante e un Ercole ${ }^{101}$, mentre per Marco Barbarese un'acquasantiera ${ }^{102}$ e per Francesco Antonio Avallone due carri e una coppia di fontane $e^{103}$.

Anche in ambito privato gli oggetti con finalità religiosa godevano di una domanda alquanto elevata, assumendo in certi casi fattezze di vere e proprie opere d'arte in miniatura, come l'acquasantiera dell'orefice Simone Parascandolo (notizie 1658-1674) ${ }^{104}$, arricchita da statuette di Sant'Anna con la Vergine Bambina in braccio, accompagnata dai Santi Giuseppe e Gioacchino, acquistata da un tal Costantino d'Abisio $^{105}$. Abbiamo, ancora, notizia di una statua della Vergine, alta 3 palmi con la base, «gettata, cicillata e lavorata» dall'orefice Antonio Monaco, remunerata il 28 luglio 1674 da Andrea de Ponte ${ }^{106}$, una sorta d'intermediario-procuratore avvezzo a mercanteggiare altresì quadri di Viviano Codazzi e Scipione Compagno ${ }^{107}$. Di particolare raffinatezza era anche l'Angelo Custode dell'oscuro Ottavio De Martino, in argento e rame indorato, posto sopra una pedagna di ebano e tartaruga con inserti similmente d'argento e rame indorato, venduta per $66 \mathrm{du}$ cati a un marchese di Dugenta, come testimonia il saldo del 4 aprile $\mathrm{I} 68 \mathrm{I}^{108}$.

Il medesimo anno si presenta particolarmente ricco di riferimenti archivistici, a cominciare dai diversi oggetti di Vinaccia acquistati dal duca di 
Valentino, come alcuni candelieri e un San Sebastiano $^{109}$. A distanza di pochi giorni Domenico del Giudice, forse dei principi di Cellamare e Giovinazzo, comperava per 40 ducati dallo sconosciuto orafo-argentiere Domenico Regi una coppia di piccole piramidi, sormontate rispettivamente da una statuetta di San Gaetano da Thiene e San Nicola di Bari, ciascuna del peso di circa 3 libbre, oltre a una bacchetta d'argento dello stesso artefice ${ }^{110}$. Invece, il 24 maggio, $\mathrm{Ni}^{-}$ cola d'Aula veniva saldato dal principe della Torella per «tutti li lavori d'argento et oro» ${ }^{111}$, ma la polizza non fornisce ulteriori dettagli.

In assenza di specifiche menzioni sull'iconografia, destinazione e committenti appare ardua la classificazione di alcune suppellettili. A questa categoria appartengono i reliquiari e le statuette che possono oscillare tra sfera pubblica e privata, adattandosi sia a una fruizione di massa sulle mense degli altari di chiese e confraternite, ma utilizzabili anche in abitazioni laiche o comunità ecclesiastiche per appagare una religiosità intimistica.
A conferma di quanto detto è quel Cristo, del valore di roo ducati, ordinato nel i665 dal cardinale Pascual de Aragón, viceré di Napoli dal i 664 al i666, tramite Cristoforo Ruiz de Pedrosa a Giacinto Porzio (notizie I6I4-1678) ${ }^{112}$, esponente della nota famiglia di argentieri ${ }^{113}$.

Tra gli altri esempi ricordiamo quel reliquiario, opera di Monte, consegnato nel i 668 a Carlo Pignatelli per una sede ignota ${ }^{114}$, o la statuetta della Vergine, commissionata nel i 673 a Ignazio Strozza da padre Innocenzo d'Arzano, francescano conventuale di San Lorenzo Maggiore di Napoli ${ }^{115}$. Ancora, se il quadro con cornice in ebano e inserti in argento di Biagio Coraggio, venduto nel i68 I a Giovanni Antonio Olivieri ${ }^{116}$, o le sei statuette con «fiori naturali» di Finelli117, volute nello stesso anno dal patrizio napoletano Giovan Battista Toc$\mathrm{co}^{118}$, parrebbero compatibili con un uso privato, nessuna ipotesi risulta possibile per le due statue dell'argentiere Giuseppe Maiorino, del peso di s libbre, acquistate nel r 682 da un certo Nicolò Fiorentino ${ }^{119}$. 


\section{Appendice documentaria}

I) Archivio Storico Banco di Napoli-Fondazione [ASB$\mathrm{Na}$ ], Banco dello Spirito Santo, giornale di cassa, 3 I agosto I 665, m. 490 , p. n. n.:

«Al p(adre) Gio(vanni) Batt(ist)a Guarino d(ucati) 30 e p(er) lui a m(ast)ro Aniello Treglia e sono a comp(imen)to di d'una testa, mani, diadema e giglio d'arg(en) to della statua di S(anto) Dom(eni)co restando d'ogni cosa sodisfatto, e p(er) esso a Vitaliano Bagnato p(er) al (trettan)ti».

2) ASBNa, Sacro Monte e Banco della Pietà, giornale di cassa, 6 luglio i668, m. 591, p. n. n.:

«A fra Serafino Spada d(ucati) ro, e p(er) lui ad Aniello Treglia argentiero d(iss)e sono a compimento di d(ucati) 50 att(es)i che li altri d(ucati) 40 l'ha ricev(u) ti con(tan)ti e p(er) n(ost)ro banco e sono in conto di d(ucati) 2 I 5.2.IO promessoli, e convenuto co(n) d(etto) An(iell)o p(er) il prezzo e fatt(ura)d'una statua de' S(an) Dom(eni)co co(n) tutte quelle clausole e cond(izio)ni posti nelli altri pag(amen)ti fatteli è sono a comp(imen) to di d(ucati) I 50: e resta dover havere d(ucati) 65.2.10, e $\mathrm{p}(\mathrm{er})$ lui a Matteo Grimaldo per alr(ettan)ti».

3) ASBNa, Sacro Monte e Banco della Pietà, giornale di cassa, I 2 dicembre I668, m. 600, p. n. n.:

«A d(on) Benedetto Giovane d(ucati) i e p(er) lui a Gio(vanni) Dom(eni)co Vinaccia disse sono in conto d'uno altare custodia e parte quali si fa fare per la cap$\mathrm{p}(\mathrm{el}) \mathrm{la}$ del S(antissi)mo nella chiesa di S(an)to Stefano di Capri in $\mathrm{n}(\mathrm{om})$ e e parte di cui $\mathrm{d}(\mathrm{ett}) \mathrm{o}$ Gio(vanni) che ne have pigliato il peso li paga».

4) ASBNa, Sacro Monte e Banco della Pietà, giornale di cassa, 20 novembre 1688, m. 900 , p. n. n.: «A don Giuseppe Spinelli duca di Laurino d(ucati) ro e p(er) lio a Gio(vanni) Dom(eni)co Vinaccia a comp(imen)to di d(ucati) 40 atteso gl'altri d(ucati) 30 l'ha ric(evu)ti p(er) lo med(esi)mo n(ost)ro b(an)co, e se li pag(a)no acciò facci fare una custodia $\mathrm{p}(\mathrm{er})$ il mon(aste)rio de $\mathrm{p}$ (adri) reformati della terra della Castelluccia con l'altre clausole poste nell'altre polize fatte $\mathrm{p}(\mathrm{er}) \mathrm{d}(\mathrm{ett}) \mathrm{a}$ causa, e $\mathrm{p}(\mathrm{er})$ lui a m(ast)ro Ant(oni)o Caputo, e sono a comp(imen)to di $\mathrm{d}$ (ucati) $28 \mathrm{p}$ (er) la custodia che sta facendo ordinatali da Gio(vanni) Cardinale intagliatore, con firma di d(ett)o Antonio Caputo».

5) ASBNa, Sacro Monte e Banco della Pietà, giornale di cassa, I 8 gennaio I666, m. 567, p. n. n.: «A d(on) Carlo De Palma reg(gen)te d(ucati) 29 e p(er) lui a Gio(vanni) Dom(eni)co Vinaccia disse a com(pimen)to di d(ucati) 75 e att(eso) gli altri d(ucati) 46 l'ha ric(evu)ti con(tan)ti in più partire, et detti $\mathrm{d}$ (ucati) 75 sono $\mathrm{p}$ (er) intiero prezzo d'una testa et mani della Beatissima Vergine e d'un puttino fatti d'ordine suo p(er) la loro chiesa di S(an)ta Maria della Pietà di Ferrara con dichiaratione che con $\mathrm{d}(\mathrm{ett})$ o pagam(en)to resta interamente soddisfatto $p(e r)$ detto lavoro, e d'ogni altra spesa fatta da lui p(er) d(ett)a causa, co(n) f(irm)a di Gio(vanni) Dom(eni)co Vinaccia».

6) ASBNa, Sacro Monte e Banco della Pietà, giornale di cassa, 26 ottobre I669, m. 6r 3, p. n. n.:

«A Franc(esco) Maria Rocco d(ucati) 76 e p(er) lui a Gen(na)ro Monte disse li paga in nome et $\mathrm{p}(\mathrm{ar}) \mathrm{te}$ del mon(aster)io di S(an) Lorenzo della Padula a comp(imen)to di d(ucati) I600, atteso l'altri i 524 li have ric(evu)ti p(er) banco et con(tan)ti et tutti sono a conto della statua d'arg(en)to che ha fatto di S(an) Bruno p(er) devotione delli padri di $\mathrm{d}(\mathrm{ett}) \mathrm{o}$ mon(aster)io con f(irm)a di Gen(na)ro Monte».

7) ASBNa, Sacro Monte e Banco della Pietà, giornale di cassa, 19 novembre 1672, m. 655 , p. n. n.: «Al d(otto)r Luise Ant(onio) Capasso d(ucati) 24 et $\mathrm{p}$ (er) lui al s(igno)r Gennaro Monte a comp(imen)to di d(ucati) Ioo atteso li altri d(ucati) $76 \mathrm{p}$ (er) detto comp(imen)to li ha da esso ric(evu)ti con(tan)ti et detti sono in conto delli $\mathrm{d}$ (ucati) 250 che si devono in n(om)e dell'un(iversi)tà del Casale di Fratta Mag(gio)re p(er) resto del prezzo di una statua di Santa Giuliana da lui da consignarsi restandoli $\mathrm{p}($ er) resto del d(etto) prezzo di d(ucati) $250 \mathrm{p}$ (er) detto comp(imen)to et $\mathrm{d}$ (etto) pagam(en)to lo fa come eletto di $\mathrm{d}$ (etta) un(iversi)tà et $\mathrm{p}($ er) lui ad Angelo de Simone $\mathrm{p}(\mathrm{er})$ altri tanti».

8) ASBNa, Sacro Monte e Banco della Pietà, giornale di cassa, Io dicembre I672, m. 655 , p. n.n.: «Al p(adre) Scipione Cacciuttolo d(ucati) 640, e p(er) lui à Lonardo Franco argentiero disse à comp(imen)to di d(ucati) I I 40, che gl'altri d(ucati) 500 l'ha ric(evu)ti; d(ucati) 400 p(er) lo b(an)co del Salv(ato)re; e gli altri d(ucati) roo = p(er) $\mathrm{n}$ (ost)ro b(an)co, e sono d(ucati) $880=$ p(er) l'intero prezzo dell'argento $\mathrm{p}$ (er) la statua di s(anto) Fran(ces)co Xaverio fatta $\mathrm{p}(\mathrm{er})$ la chiesa della casa professa, e gli altri $\mathrm{d}$ (ucati) 252 = sono in conto della fattura di d(etta) statua con firma di d(etto) Lonardo Franco».

9) ASBNa, Sacro Monte e Banco della Pietà, giornale di cassa, 28 marzo I673, m. 665, p. n. n.:

«A p(adre) d(on) Michele Caracciolo d(ucati) r 20 e p(er) lui a Lonardo Franco argentiero, disse esserno a camp(imen)to di d(ucati) 520 che l'altri l'ha ricevuti in diverse volte et banchi in conto di d(ucati) 530 prezzo d'una statua a mezza busta d'argento con pedagna di legno rinargentato da esso fatta del glorioso S(an) Gios(epp)e $\mathrm{p}(\mathrm{er})$ sertitio d'una signora monaca nella città di Barletta et de comessione di $\mathrm{d}($ ett)o [...] $\mathrm{p}$ (adre) $\mathrm{d}($ on) Michele e p(er) lui ad Ang(el)o de Simone p(er) al(trettant)i».

Io) ASBNa, Banco di Sant'Eligio, giornale di cassa, I I agosto $1673, \mathrm{~m} .387$, p. n. n.: 
«Ad Aniello e Onofrio de Vivo d(ucati) i 2, e p(er) loro a Carlo Marante e Fabritio Giordano proc(urato)ri del glorioso S(an)to Trifone della terra di Tramonti del luogo di Cesarano e sono $\mathrm{p}(\mathrm{er})$ tanti che il $\mathrm{q}($ uonda)m Dom(eni)co de Vivo loro pr(ocurato)re nel suo ult(imo) tes(tamen)to lasciò titolo legati à d(et)to glorioso s(an) to $\operatorname{co}(\mathrm{n})$ cond(izio)ne che questi s'havessere à impiegare nella compra d'una statua d'argento del d(ett)to glorioso $\mathrm{S}($ an)to Trifone e $\mathrm{d}$ (ett)to pag(amen)to lo farà all'hora quando sarà complita detta statua e per loro a Dom(eni) co de Gregorio p(er) saldo, e comp(imen)to dell'intero prezzo della statua di $\mathrm{d}(\mathrm{et})$ to glorioso s(an)to Trifone $q($ ues)to $\mathrm{p}($ rese)nte consignateli $\mathrm{p}(\mathrm{er})$ uso di $\mathrm{d}(\mathrm{ett})$ a parrocchiale Chiesa di $S$ (anta) $M($ ari)a di Cesarano della terra di Tramonti e p(er) esso Dom(eni)co d(ett)a statua promessa di fare, come dalla par(ti)ta di n(ost)ro $\mathrm{b}(\mathrm{an}) \mathrm{co}$ delli 9 agosto dell'anno caduto $1672[\ldots]$ e co(n) d(ett)o pag(amen)to d(ett)o Dom(eni)co no(n) resta à conseg(ui)re cos'altra att(es)o l'altra quantità $\mathrm{p}(\mathrm{er})$ il comp(letamen)to di d(ett)a statua si sono pag(a)ti parte al q(uonda)m Aniello Treglia con(tan)ti, e parte à d(ett)o Dom(eni)co de Greg(orio) parte con(tan)ti, e parte p(er) il med(esimo) n(ost)ro b(anc)o, co(n) sua f(irm)a a pie».

I I) ASBNa, Sacro Monte e Banco dei Poveri, giornale di cassa, 16 febbraio 1674 , m. 492, p. n. n.:

«A d(on) Fran(ces)co de Vigliena d(ucati) Ioo, et per lui a $\mathrm{p}$ (adre) maestro Ignatio Genusio dell'ord(in)e de $\mathrm{p}$ (adri) $\mathrm{p}$ (redicatori) de pagarli di ord(in)e et mandato di d(on) Antonio Genusio suo nepote della suma esatta dal sig(no)r And(re)a Cavoti in virtù d'istrum(en)to di transatt(io)ne, quali d(ucati) roo resti tenuto et obligato sempre detto $\mathrm{p}$ (adre) maestro farceli bonificare da d(etto) d(on) Andrea o ristituirli subbito, et p(er) esso a Vincenzo di Urso argentiero, et esserno a complim(en)to di d(ucati) 200, atteso l'altri d(ucati) roo l'ha ricevuti di contanti, et detti d(ucati) 200 sono in conto di una statua di argento, che il d(ett)o Vin(cen)zo fa del beato Pio Quialti [Pio Quinto], et p(er) esso a Nicola Maramaldo a complim(en)to di d(ucati) 200, che l'altri d(ucati) Ioo p(er) detto complim(en)to l'ha da esso ricevuti contanti, $\mathrm{d}$ (iss)e $\mathrm{p}$ (er) conto dell'argento, et manifattura di un mezzo ... [omissione nel testo] di piangia d'argento senza però testa, et mani promessili fare del beato papa Pio V, una con il suo camauro similm(en)te di piangia di argento in frà un mese $\mathrm{d}($ ett)o mezzo beato, et infra mesi due $\mathrm{d}(\mathrm{ett}) \mathrm{o}$ camauro dalli $\mathrm{I} 3 \mathrm{~d}$ (ett)o corr(en) te mese di feb(bra)io havanti memorandi come q(ue)sto et altro s'innarra il p(rese)nte di fra di loro fatto, et a loro futura cau(te)la relassato in potere di $\mathrm{n}(\mathrm{ota})$ ro Aniello di Luise di Napoli a lui si refe et quatenus di bisogno fusse s'habbia relatione, et per esso Nicola per mano di n(ota)ro Darese d'Angelo in curia di n(otar)e Carl'Antonio d'Urso di Napoli, a lui ducati ıoo».

I 2) ASBNa, Banco dello Spirito Santo, giornale di cassa, I7 ottobre I680, m. 6I 2, p. 473:

«A Dom(eni)co Pavone d(ucati) I 8 et $\mathrm{p}($ er) lui al $\mathrm{p}$ (adre) d(on) Fran(ces)co Borgia celestino e sono p(er) il $\operatorname{prez}(\mathrm{z}) \mathrm{o}$ d'una statua a mez(z)o busto cho hava fatto fare di S(an) Pietro Celestino a Majella d'ord(i)ne della s(igno) ra prin(cipes)sa di $\mathrm{S}(\mathrm{an})$ to Buono, et a lui consig(na)to $\mathrm{p}(\mathrm{er})$ mandarla a d(et)ta sua s(igno)ra et p(er) esso à f(ra) Dom(eni)co d'Agostino p(er) alt(rettan)ti».

I3) ASBNa, Sacro Monte e Banco della Pietà, giornale di cassa, 29 gennaio $\mathrm{I} 68 \mathrm{I}, \mathrm{m} .780$, p. n. n.:

«A d(onna) Beatrice Ventimiglia mar(che)sa di S(an) Giorgio d(ucati) roo e p(er) lei a Gio(vanni) Dom(eni)co Vinaccia e se li pagano in conto della statua d'arg(ento) della gloriosa S(ant')Anna de q(ua)le ha pigliato peso de farla, e consignarla p(er) li io del mese di mag(gi)o p(rossi)mo venturo di tutta perfett(io)ne, qualità e bontà, con che p(er) la fattura d'essa se l'habbiano da lui a pagare d(ucati) I 80 includendosi in quello il modello, politura et ogni altra cosa concorrentino alla totale perfettione di d(etta) statua e perche no(n) aveda il peso di libre 50, e tutto il di più del prezzo di detta statua se li pagherà cioè d(ucati) 300 nel p(ri)mo del entrante mese di marzo, e li restanti in tempo li consignara la statua pred(ett)a, quali no(n) consignandoli nel sud(dett)o tempo dieci di mag(gi)o sia a suo arbitrio, o farla fare da altra persona, sul $\mathrm{q}(\mathrm{u})$ ale caso sia obligato d(etto) Gio(vanni) Dom(eni) co alla restitu(tio)ne di tutta quella quantità di denari, che in quel tempo da lei si ritroveranno pagati p(er) la causa pred(ett)a una con tutti danni, spese et interessi che $\mathrm{p}(\mathrm{er})$ la causa predetta li preveniranno, tanto $\mathrm{p}(\mathrm{er})$ li denari ritenuti in suo potere, quanto per ogni altra causa [...], quale pagamento lo fa come madre, balia e tutrice di d(on) Carlo Ant(onio) Tocco Principe d'Acaia, e $\mathrm{m}$ (arche)se di $\mathrm{M}(\mathrm{on})$ te $\mathrm{m}(\mathrm{i})$ letto suo figlio, e p(er) ademp(imen)to del voto fatto dal d(ett)o suo figlio alla gloriosa S(ant')Anna $\mathrm{p}(\mathrm{er}) \mathrm{mag}(\mathrm{gio}) \mathrm{re}$ sua veneratione $\mathrm{p}(\mathrm{er})$ la devotione, che ne tiene in tempo, che hebbe l'infermità delle bone acciò recuperarle come recuperò la salute. Co(n) f(irm)a di d(etto) Gio(vanni) Dom(eni)co».

I4) ASBNa, Banco dell'Annunziata, giornale di cassa, Io febbraio I68 I, m. 547, p. 67:

«A d(onna) Margherita de' Rossa d(ucati) roo e pe(r) essa a Genn(a)ro Monte et sono in co(n)to della nuova statua d'arge(n)to di S(an)ta Fortunata che doverà fare proportionata e d'ogni perfett(io)ne sop(r)a la pedagna d'arge(n)to da essa consignateli p(er) d(etto) effetto come anco li ha consignato la statua vecchia libro palma e corona d'arge(n)to di d(etta) Santa quale statua libro palma e corona d(etto) Genn(a)ro [...] e notare il peso di d(etto) argento, e poi squagliare d(etto) Arge(n)to p(er) rifare la nuova statua sop(ra) la d(etta) pedagna e promette $\mathrm{d}(\mathrm{ett}) \mathrm{o}$ Genn(a)ro: $d(e t t) a$ nuova statua consignarla finita e di tutta perfett(io)ne $\mathrm{p}(\mathrm{er})$ tanto il mese di giug(no) I68 I co(n) ta(n)ti a d(ett)o Genn(a)ro Monte con sua firma».

i 5) ASBNa, Sacro Monte e Banco della Pietà, giornale di cassa, 9 giugno $168 \mathrm{I}, \mathrm{m} .777$, p. n. n.:

«A d(on) Paolo Carafa vescovo d'Aversa d(ucati) 200 e p(er) lui a Gio(vanni) Dom(eni)co Vinaccia a conto della statua d'argento de San Paolo Apostolo che sta facendo e $\mathrm{p}(\mathrm{er})$ lui a Gaetano Solombrino $\mathrm{p}(\mathrm{er})$ al(tret)tanti». 
I6) ASBNa, Banco di San Giacomo, giornale di cassa, i 8 dicembre I68 I, m. 427, p. 535 :

"A Cesare Maffei d(ucati) ro e p(er) esso al p(adre) Luiggi Maffeo p(er) al(trettan)ti e p(er) esso a Gio(vanni) Dom(eni)co Vinaccia a comp(imen)to de ducati 60.3 atteso li altri d(ucati) 50.3 li ha ric(evu)ti in diverse volte si per b(an)co come de con(tan)ti [...] e di d(ucati) 60.3 sono li conto del lavoro dell'arg(en)to della statua che sta facendo p(er) il serv(iti)o della sua chiesa della Cong(regazio)ne dell'Oratorio de Nap(oli) e p(er) esso a Genn(ar)o D’Errera p(er) al(trettant)i».

I7) ASBNa, Banco dell'Annunziata, giornale di cassa, I6 settembre I666, m. 402, p. n. n.:

«A d(on) Innocentio de Forte d(ucati) 4 I 6.3 e p(er) esso a Fra(nces)co Califano orefice e sono a comp(imen) to di d(ucati) 626.3 atteso gli altri d(ucati) 2 ro li ha ri$\mathrm{c}(\mathrm{evu}) \mathrm{ti}$ in due partite $\mathrm{p}(\mathrm{er})$ il medesimo nostro banco, $\mathrm{q}$ (ues)ti d(ucati) 626.3 sono $\mathrm{p}$ (er) l'intiero prezzo di uno panno o pallio di argento dell'altare maggiore della chiesa della madonna di Piedigrotta, da esso consig(na) te $\mathrm{q}$ (ues)ti $\mathrm{d}$ (ucati) 626.3 sono cioè $\mathrm{d}$ (ucati) $\mathrm{I} 60 \mathrm{p}(\mathrm{er})$ la fattura dello panno seù pallio secondo l'accordio et conventione havuta con d(ett)o Fra(nces)co della q(ua)le se ne fa mentione nelle $\mathrm{p}$ (assat)e partite di pagam(en)to fattoli p(er) il nostro banco [...] e p(er) esso a Gius(epp)e Califano $\mathrm{p}(\mathrm{er}) \mathrm{Al}(\operatorname{trettant}) \mathrm{i}$.

I 8) ASBNa, Banco di Sant'Eligio, giornale di cassa, I4 luglio I672, m. 379, p. n. n.:

«A d(on) Gio(vanni) Pietro Marana d(ucati) 200 e p(er) lui a Gio(vanni) Dom(eni)co Vinaccia, e sono in conto di d(ucati) 300 intiero prezzo di uno panno d'altare d'arg(en) to traforato e corniciato di peso di libre 18 in conformità del disegno fatto dal med(esimo) Gio(vanni) Dom(eni)co che se ne cons(er)va uno da lui e l'altro da d(ett)o Vinaccia firmato da lui e da esso Gio(vanni) Dom(eni)co di palmi 9 di lunghezza avantagiati e palmi 4 di larghezza di tutto di buona qualità et à lode d'esperti q(ua)le panno d'arg(en)to del modo e qualità ut s(upr)a il d(ett)o Gio(vanni) Dom(eni)co si e tenuto et obligato a consignarlo qui in Napoli frà mesi da $\mathrm{I} 2[\ldots]$ che $\mathrm{d}$ (etto) panno d'arg(ento) servirà p(er) l'altare della cap(pel)la del S(antissimo) Sac(rament) o della chiesa parr(occhia)le della città di Modugno e lui fa il p(rese)nte pag(amen)to di d(ucati) 200 di suoi propri denari p(er) altrecause sua mente movenino; e p(er) esso à Giose(ppe) Alfano p(er) altri(tanti)».

I9) ASBNa, Banco di Sant'Eligio, giornale di cassa, 2 I giugno 1673 , m. 385 , p. n. n.:

«A Carlo De Mari principe d'Acquaviva d(ucati) I 88.3. Io e p(er) lui a Giou(sepp)e d'Auria argentiero a comp(imen)to di d(ucati) 200 att(es)o l'altri d(ucati) I I.I.I l'ha ricevuto in tanto argento, e tutti detti d(ucat) i 200 sono a comp(imen)to di d(ucati) 600 che l'altri d(ucati) 400 ce l'ha pagati $\mathrm{p}($ er) il banco di S(an) Giac(om)o a 6 aprile pross(im)o pass(a)to et in c(on)to d'un palio di argento che stà facendo in tutto come si dichiara in $\mathrm{d}(\mathrm{ett})$ a polizza de d(ucati) $400 \mathrm{co}(\mathrm{n})$ sua f(irm)a a lui $[\ldots]$ ».
20) ASBNa, Sacro Monte e Banco della Pietà, giornale di cassa, 23 giugno 1673 , m. 665, p. n. n.:

«A Prospero Sebastiano d(ucati) 200 e p(er) lui a Lonardo de Franco argentiero disse esserno a comp(imen) to di d(ucati) I 200, atteso l'altri d(ucati) I000 l'ha ricevuti $\mathrm{p}$ (er) il medesimo nostro Banco e disse esserno in conto della fattura dell'argento del paliotto e candelieri che si è obligato fare $\mathrm{p}(\mathrm{er})$ servitio di $\mathrm{d}(\mathrm{on})$ Mauro Cesarini, abb(at)e di Montecasino med(ian)te instru(mento) $\mathrm{p}($ er) mano di n(ota)r Dom(enic)o Cardamomo di Nap(oli) al quale [...] e p(er) lui ad Ang(el)o de Simone $\mathrm{p}(\mathrm{er})$ altritanti».

2 I) ASBNa, Sacro Monte e Banco della Pietà, giornale di cassa, 24 marzo I682, m. 796, p. n. n.:

«Al p(adre) Nicola de Madaluni d(uca)ti 40 e p(er) lui al p(adre) Gen(na)ro Conte p(er) a(ltrettan)ti, e $\mathrm{p}$ (er) lui a Matteo Treglia argentiero, cioè d(ucati) 39 à comp(imen)to di d(ucati) 59, att(es)o l'altri d(ucati) 20 l'ha ric(evu)ti da lui a 4 dec(embre) i68 I co(n) f(ed)e di credito in testa ma p(er) lo b(an)co di S(anto) Eligio, e li car(li)ni io restanti di q(ues)ta $\mathrm{p}(\mathrm{re})$ sente $\mathrm{f}(\mathrm{ed}) \mathrm{e} \mathrm{d}(\mathrm{ett}) \mathrm{o}$ Matteo ce li ha rest(itui)ti con(tan)ti, e di d(ucati) 59 sono cioè d(ucati) 4I p(er) prezzo, et peso d'arg(en)to [...] che sono andate et hà poste $\operatorname{co}(\mathrm{n})$ una figura d'arg(en) to di S(an) Pietro martire, fatta lavorare da d(ett)o Matteo, et à lui conseg(na)ta et posta nel mezo d'un panno d'altare ricamato fatto fare da esso girante $\mathrm{p}$ (er) serv(iti) o et ornam(en)to dell'altare magg(io)re della loro chiesa di S(an) Pietro martire, e li restanti d(ucati) i 8 ce li paga $\mathrm{p}(\mathrm{er})$ tutte le spese, et manifatture da esso fatte nella d(ett)a figura d'arg(en)to di d(ett)o S(an) Pietro, restando con $\mathrm{d}(\mathrm{ett}) \mathrm{o}$ pagam(en)to intieram(en)te da lui sodis(fa)to tanto $\mathrm{p}(\mathrm{er})$ causa di $\mathrm{d}(\mathrm{ett})$ a figura, quanto $\mathrm{p}($ er) quals(iasi)a altra causa, con dichiarat(io)ne no(n) dovere da lui conseguire altra nuvena di denaro $\mathrm{p}(\mathrm{er})$ quals(iasi)a altra causa, e p(er) lui a Gen(na)ro Scotto $\mathrm{p}$ (er) a(ltrettan)ti, et $\mathrm{p}$ (er) lui a Gio(vanni) Dom(enico) Farina $\mathrm{p}($ er) a(ltrettan)ti».

22) ASBNa, Banco dello Spirito Santo, giornale di cassa, 5 settembre I682, m. 628, p. I 5 :

«Al p(adre) m(aest)ro fra' Dom(eni)co M(ari)a Marchesi d(ucati) so et p(er) lui a Gio(vanni) Dom(eni) co Vinaccia e sono a com(pimen)to di d(ucati) roo atteso gli altri d(ucati) so l'hà ricevuti de contanti e sono in conto di d(ucati) 400 che li paga $\mathrm{p}$ (er) peso d'argento e fattura del panno d'altare dell'altare mag(gio)re della chiesa loro di S(an) Dom(eni)co di q(uest)a città secondo il disegno e patto tra loro convenuti e $\mathrm{p}(\mathrm{er})$ esso a Gio(vanni) Dom(eni)co Favino p(er) al(trettan)ti».

23) ASBNa, Sacro Monte e Banco della Pietà, giornale di cassa, 20 giugno I68 I, m. 778, p. n. n.: «Al p(adre) Dom(eni)co M(ari)a Marchesi d(ucati) roo, et p(er) lui a Gio(vanni) Dom(eni)co Vinaccia in co(n)to della custodia d'arg(en)to della chiesa di S(an) Tomaso secondo li patti tra loro con(venu)ti e p(er) lui a Gaet(an)o Solombrino $\mathrm{p}(\mathrm{er})$ al (trettant)i». 
24) ASBNa, Banco dello Spirito Santo, giornale di cassa, 9 ottobre 1687, m. 673 , p. 265 :

«A sor M(ari)a Rosolea Mercurio d(ucati) 250 e p(er) lei a Nicola Aula orefice disse sono in conto del prezzo di candelieri d'arg(en)to sei di peso libbre dieci incirca $\mathrm{p}(\mathrm{er})$ ciascuno et alti palmi q(ua)ttro scarsi che il detto Nicola ha promesso, e s'è obbligato manufatturare e consegnarli d'ogni qualità e perfet(tio)ne co(n) il solito Marco [...] d'esso Nicola come de consoli dell'Arte d'Argentieri p(er) netto l'ultimo aprile $\mathrm{r} 688$ e dell'istesso modo e forma co(n) li stessi lavori co(n)forme li candelieri d'Arg(en)to novam(ente) fatti dal v(enerabi)le mon(aste)rio di S(an) Pietro a Maiella et questi a rag(gio) ne de $\mathrm{d}$ (ucati) I3.r.Io la libra inclusa la manifattura et ogni altra spesa, e che nella pedagna di ciascuno d'essi candelieri ci sia d'una parte scolpita l'imagine della SS(antissi)ma Concett(ion)e cola coronella in testa indorata [...] altra lamia di sua casata, e dall'altra il suo nome scolpito distante suor M(ari)a Rosolea Mercurio e co(n) li patti e promesse conf(orm)e il netto più largam(en)te si conviene nello Is(trumen)to a 4 del cor(rent)e rog(at)o p(er) m(an)o di n(otai)o cano(ni)co Ant(on)io Cannatelli di Napoli al quale in Omnibus s'hab(bi)a rela(tio)ne, et $\mathrm{p}(\mathrm{er})$ esso a Salvatore Nicolo p(er) al(trettan)ti».

25) ASBNa, Banco di Sant'Eligio, giornale di cassa, 22 dicembre 1673 , m. 388 , p. n. n.:

«Al monast(e)rio di S(anto) Andrea delle Monache d(ucati) 94.I.I7 co(n) f(irm)a di sore Chiara Maria di Bologna priora in $\mathrm{d}$ (ett)o monas(te)ro a Nicola d'Auria orefice argentiero $\mathrm{p}(\mathrm{er})$ saldo e final pag(amen)to cossì della valuta seu prezzo d'una croce d'argento grande con il monte servita $\mathrm{p}(\mathrm{er})$ l'altare mag(gio)re di loro chiesa come $\mathrm{p}$ (er) la fattura di essa cioè $\mathrm{d}$ (ucati) IO2I.3.I7 prezzo di libre 99 et once $2 / \mathrm{I} / 2$ d'argento a d(ucati) Io e carlini 3 la libra e d(ucati) 600 p(er) la fattura di essa Croce, att(es)o li altri d(ucati) i 527.2 a comp(imen)to di $\mathrm{d}$ (ucati) I62 I.3.I7 d(et)to Nicola l'ha ricevuti così p(er) diverse partite di banchi come $\mathrm{p}(\mathrm{er})$ contanti la quale Croce co(n) d(etto) monte è stata a loro consig(na)ta e ricevuta da d(ett)o Auria itache con d(ett)o pagam(en)to resta da loro interam(en)to sodisfatto, così $\mathrm{p}(\mathrm{er}) \mathrm{d}(\mathrm{ett}) \mathrm{o}$ prezzo di esso argento come $\mathrm{p}(\mathrm{er})$ la fattura no(n) restando à conseguire altro $\mathrm{p}(\mathrm{er})$ detta Causa e $\mathrm{p}(\mathrm{er})$ lui a Fran(ces)co Califano p(er) alt(rettant)i».

26) ASBNa, Banco dello Spirito Santo, giornale di cassa, 20 maggio I 682, m. 623, p. 444 (879-880):

«All'abb(a)te Gen(na)ro Filomarino d(ucati) 20, et p(er) lui a Gio(vanni) Dom(eni)co Vinaccia in conto d'una Croce d'arg(en)to che l'ha lavorato, et consegnato p(er) la chiesa di S(an)ta M(ari)a del Monte della Terra di Tiggiano; et p(er) esso a Fran(ces)co Caputo p(er) alt(rettant)i».

27) ASBNa, Banco dello Spirito Santo, giornale di cassa, 28 luglio I683, m. 63 I, p. I I 50:

«Al(l')Ab(at)e Gen(na)ro Filomarino d(ucati) 22.2.9 $\mathrm{p}(\mathrm{er})$ lui a Dom(eni)co Vinaccia a comp(imen)to di d(ucati) $659 \mathrm{~g}($ ran)a $9 \mathrm{t}$ (ari) 3.I 5 att(es)o l'altri res(tan)ti $\mathrm{d}$ (ucati) 637.16.24 ric(evu)ti da esso cioè d(ucati) 496.7.6 $\mathrm{p}(\mathrm{er})$ lib(br)e 48 et oncia una di 6 candelieri vecchi d'argento consignateli nel mese di gennaro 1683 a d(ucati) IO.I.I la lib(br)a et d(ucati) I 42 in più volte $\mathrm{p}(\mathrm{er})$ diversi banchi et d(ucati) 659.3. Is sono p(er) l'intiero prezzo di 6 candelieri d'argento che l'ha lavorato et consig(na)to di lib(br)e so et oncie 9 per la chiesa delle monache della SS(antissi)ma Trinità di $\mathrm{q}($ ue)sta città a $\mathrm{d}$ (ucati) I 3 la lib(br)a tra argento e fattura dichi(aran)do che con detto pagam(en)to resta da esso et da $\mathrm{d}$ (etto) mon(aste)rio sodisfatto non rest(an)do a conseg(ui)re cos'alcuna per esso a Gio(vanni) Dom(eni)co Farina p(er) alt(rettanti)».

28) ASBNa, Banco di San Giacomo, giornale di cassa, 30 gennaio 1675 , m. 382 , p. 108:

«A fra Martino di Napoli camaldolese d(ucati) roo e p(er) esso a Gio(vanni) Dom(eni)co Vinaccia d(iss)e li paga in nome e de p(rop)rj denari del s(acro) eramo de camaldoli di Nola e sono cioè d(ucati) 4I.4.5 a comp(imen)to di d(ucati) 552 atteso d(ucati) 5 Io.I 5 l'ha ric(evu)ti da esso il $3 \mathrm{~d}$ (ucati) roo sotto sua per il $\mathrm{b}$ (an) co dei Poveri ad ottob(r)e 1674 et l'altri con(tan)ti e tutti d(ett)i d(ucati) 552 sono p(er) l'intiero p(re)zzo di sei candelieri d'argento e loro manifatt(u)ra conf(orm)e la conven(zio)ne havuta fra di loro et l'altri d(ucati) i 8 sono in conto di d(ucati) ror.22 intiero prezzo di una custodia c(on) puttini gradini avanti l'altare piedistalle giarre et candelieri di legname ingessate pittate e parte argen(ta)te una cola portella di d(ett)a custodia d'argento di peso d'una libra e sua manifatt(u)ra co(n) altre spese da lui fatte $\mathrm{p}(\mathrm{er})$ le tutte cause e $\mathrm{p}(\mathrm{er})$ esso ad Angelo de Simone p(er) al(trettant)i».

29) ASBNa, Banco di San Giacomo, giornale di cassa, 27 marzo I675, m. 382, p. 344:

«A d(on) Pietro Pesule d(ucati) 60 e p(er) esso a Lonardo de Franco argentiero de Nap(oli), q(ua)li sono al total complitam(en)to delli d(ucati) i 80 che li doveva $\mathrm{p}(\mathrm{er})$ la fattura della custodia fatta a S(an) Nicola di Bari comessali dal can(oni)co d(on) Paulo Cardeani custode del sacro altare dando $\mathrm{p}(\mathrm{er})$ nulli tutti li conti che siano insino al tempo de 19 di marzo cor(ren)te si da' una parte come dell'altra che si potessero toccare includendovi anco le part(i)te di banco e p(er) esso a Fran(ces)co Ant(oni)o Antrilone p(er) alt(rettan)ti».

30) ASBNa, Banco del Salvatore, giornale di cassa, i I marzo I673, m. I 82, p. n. n.:

«A Dionisio Lazzeri d(ucati) is e p(er) lui a Dom(eni) co Marinello disse $\mathrm{p}(\mathrm{er})$ uno basso rilievo di rama indorato fatto $\mathrm{p}(\mathrm{er}) \operatorname{serv}(\mathrm{iti}) \mathrm{o}$ alla custodia $\mathrm{p}$ (er) l'altare della $\mathrm{S}$ (antissi)ma Ann(unzia)ta della città di Gaeta e p(er) lui a Mariano Gentile p(er) alt(rettant)i».

3I) ASBNa, Banco di San Giacomo, giornale di cassa, 2 I aprile $\mathrm{I} 674, \mathrm{~m} .374$, p. n. n.:

«Al padre Gio(vanni) Batt(ist)a Casilei d(ucati) 45 e p(er) esso a Gio(vanni) Dom(eni)co Vinaccia a complimen- 
to di d(ucati) 550 atteso li d(ucati) 300 l'ha haverà da esso nostro banco e d(ucati) 250 contanti e d(ett)i d(ucati) 550 sono in conto del prezzo del peso d'argento che sarà necessario $\mathrm{p}(\mathrm{er})$ un baldacchino che sta lavorando $\mathrm{p}(\mathrm{er})$ servizio della della chiesa della casa professa del Gesù rimettendosi $\mathrm{d}$ (etto) Vinaccia ad esso p(er) la fattura di d(ett)a spesa e $\mathrm{p}($ er) esso al d(etto) de Simone $\mathrm{p}($ er) alt(rettant)i».

32) ASBNa, Sacro Monte e Banco della Pietà, giornale di cassa, Io marzo I68 I, m. 778 , p. n. n.:

«A d(on) Gio(vanni) Bifolco d(ucati) Ioo, e p(er) lui a d(onn)a Vittoria e, d(onn)a Maria Coppola p(er) un pronto gratioso che lui li fa $\mathrm{p}(\mathrm{er})$ doverceli restituire alla fine di luglio I68I, et p(er) loro ad And(re)a Finelli à comp(imen)to di d(ucati) 500 [...], atteso l'altri d(ucati) 450 l'ha ric(evu)ti p(er) par(ti)te de diversi banchi, e con(tan)ti e di d(ucati) 550 sono p(er) il prezzo dell'argento e manifattura d'un ostensorio, overo baldacchino p(er) il S(antissi)mo Sacram(en)to da esso And(re)a manifatturato, et a loro conseg(na)to di loro intiera sodisfa(tio) ne, e con d(ett)o pagam(en)to; d(ett)o And(re)a resta intieram(en)te sodisfatto $\mathrm{p}(\mathrm{er})$ tutti li conti [...]. In piè co(n) f(irm)a di d(ett)o And(re)a Finelli».

33) ASBNa, Banco dell'Annunziata, giornale di cassa, 30 giugno $1672, \mathrm{~m} .456$, p. n. n.:

«A d(on) Carlo Pignatelli d(ucati) roo e p(er) esso a Gennaro Monte disse sono in conto della spesa e lavoro d'un baldachino di rame indorato e d'argento che sta facendo p(er) serv(iti)o della chiesa di S(an)ta Maria della Sapienza di questa città, e disse pagarli de proprj denari e d'ord(in)e e della s(igno)ra Sor Maria Fortunata Caracciola monica del med(esim)o monast(er)o e p(er) esso ad Angelo de Simone p(er) alt(rettanti)».

34) ASBNa, Banco del Popolo, giornale di cassa, 5 dicembre I665, m. 376, p. 309-310:

«A d(on) Angelo Ant(oni)o de Conforte d(ucati) I 9 e $\mathrm{p}(\mathrm{er})$ esso a Jac(op)o And(re)a Perrore $\mathrm{p}(\mathrm{er})$ al(tretten)ti, e p(er) esso a Lenardo de Franco argentiere d(iss)e pagarli in nome e parte d(onna) Violante di Sangro monaca del $\mathrm{m}$ (onaste)rio di $\mathrm{S}$ (anto) Gaudioso di questa città $\mathrm{d}$ (iss)e a comp(imen)to di d(ucati) I346.IO, atteso li altri li ha ric(evu)ti parte $\mathrm{p}(\mathrm{er}) \mathrm{p}$ (artit)e di b(anch)i e parte con(tan) ti [..] d(ucati) I346.10 sono cioè $\mathrm{d}$ (ucati) 846.10 di prezzo dell'argento di due sgabelli co(n) due statue di argento et una gradina o piedi stalli fatto all'altare di $\mathrm{S}$ (anta) Maria d'Agnone in d(ett)o mon(aste)rio similare de argento et li altri d(ucati) 500 sono p(er) spese fattura et ogni altra cosa ove potere pretendere $p(e r) d(e t t i)$ sgabelli e gradini co(n) piedi stalli dichiarando che co(n) questo pagam(en)to d(et)to Lonardo resta intieram(en) te sodisfatto tanto dell'argento manifattura et ogni altra spesa cosa di d(ett)o argento lavorato p(er) ser(vi)tio di $\mathrm{d}(\mathrm{ett}) \mathrm{a}$ Violante in $\mathrm{d}(\mathrm{et})$ to monasterio di S(ant)o Gaudioso quanto $\mathrm{p}(\mathrm{er})$ ogni altra sua pretende(n)za».

35) ASBNa, Banco dell'Annunziata, giornale di cassa, 20 dicembre 1669 , m. 430 , p. n. n.:
«A fra Pauolo de' Montesarchio d(ucati) is e p(er) esso a Genn(a)ro Monte argentiero a compim(en)to di d(ucati) 90 att(es)o l'altri l'ha ric(evu)ti di cont(an) ti e d(ucati) 90 sono in conto di d(ucati) 340 che deve conseg(na)re dalla ven(erabi)le cappella del b(ea)to Giacomo della Marca p(er) fattura di due angeli d'arg(en)to dichiarando che d(ett)o s(igno)r Genn(a)ro tanto della fattura q(uan)to dell'arg(en)to del quale è stato sodisfatto resta cred(ito)re dà d(ett)a capp(el)la in d(ucati) 250. È p(er) esso a Ger(oni)mo Simone p(er) al(trettan)ti».

36) ASBNa, Banco dell'Annunziata, giornale di cassa, 20 febbraio I67I, m. 447, p. n. n.:

«A Gio(vanni) Bat(tis)ta e Vinc(enz)o Mezzamonaco $\mathrm{d}$ (ucati) io e p(er) essi al r(everen)do p(ad)re fra Paulo de Montesarchio sacristano della cappella del glorioso beato Giacomo della Marca sita dentro la real chiesa di S(an)ta Maria della Nova; questi se li donano elemositatili p(er) questa volta tanta, e p(er) esso a Genn(aro) Monte argentiero a comp(imen)to di d(ucati) I Io, atte(s) o l'altri l'ha ric(evu)ti de cont(an)ti, e d(ucati) i io se li pagano per ult(im)o e final pagam(en)to di d(ucati) 650 che doveva conseg(na)re p(er) fattura di due angeli d'argento fatti nella ven(erabi)le cappella del beato Giacomo della Marca dichiarando che d(etto) s(igno)r Genn(ar)o Monte resta apieno, et intieram(en)te sodisfatto da detta cappella tanto dell'argento quanto della manifattura di $\mathrm{d}(\mathrm{ett}) \mathrm{i}$ angeli in modo che no(n) tiene preventione alc(un)a in d(ett)a cappella p(er) $d(e t t) i$ angeli, e co(n) d(ett)o pagam(en)to no(n) resta a conseg(ui) re cos'alc(un)a di tutto, e' quals(ivogli)a arg(en)to che hà fatto $\mathrm{p}(\mathrm{er})$ insino a $\mathrm{d}(\mathrm{etta})$ giornata 23 dicembre 1670 , e' p(er) esso ad Angelo de Simone p(er) altri tanti».

37) ASBNa, Banco dello Spirito Santo, giornale di cassa, 19 ottobre 1671, m. 538, p. 219 (439):

«A fra Bonifacio de Nap(oli) d(ucati) 40 et p(er) lui a Nicola d'Aula et sono a complim(en)to di d(ucati) Iso, att(es)e l'altri d(ucati) roo ce l'ha dati con(tan)ti, in virtù d'Istr(ument)o per $\mathrm{m}$ (an)o di $\mathrm{n}$ (ota)r Hippolito Marullo, et l'altri d(ucati) Io con(tan)ti, et sono in conto dell'arg(en)to tanto delle cornice have da fare allo baldacchino della cappella di S(ant)a M(ari)a della Gratia dentro S(ant)a M(ari)a della Nova, come da d(ett) o istr(ument)o per m(an)o di d(et)to $\mathrm{n}$ (ota)r Hippolito Marullo di Nap(oli), q(uest)i in conto delli candelieri haverà da fare anco per $\mathrm{d}($ ett) a cappella, et per esso ad Angelo de Simone $\mathrm{p}(\mathrm{er})$ alt(rettant)i».

38) ASBNa, Banco di San Giacomo, giornale di cassa, 30 gennaio I675, m. 382 , p. I I 4:

«A Mario Luini d(ucati) 2 ro e p(er) esso ad Antonio Monaco d(iss)e sono a com(pimen)to di d(ucati) 740 atteso l'altro d(ucati) 530 l'hà ricev(u)ti cioè $\mathrm{d}$ (ucati) 500 $\mathrm{da}$ esso in div(ers)e par(tit)e di banco e d(ucati) 30 in cont(an)ti da d(on) Carlo Grimaldi et tutti sono p(er) $\mathrm{u}(\mathrm{pe}) \mathrm{ra}$ di baldacchino d'argento e rame indorato fabricato da d(ett)o Antonio p(er) la Madonna SS(antissi) ma del Carmine di Nap(oli) d'ord(in)e di d(ett)o Carlo, 
il quale baldacchino è stato di peso lib(re) 34 et once 2 che a d(ucati) IO.I.Io la lib(r)a ascendono a d(ucati) 35 I.4.Io e la fattura stimata co(n) il rame et oro da Tomase Cavallo e Gio(vanni) Dom(eni)co Vinaccia periti eletti di comun conventio tra d(et)to Monaco e D(on) Filippo Grimaldi Principe di Gerace fratello di d(etto) $\mathrm{D}$ (on) Carlo ascendere d(ucati) 405 che tutto importano $\mathrm{d}$ (ucati) 756.4.10 che il dipiù $\mathrm{d}$ (ucati) I6.410 il $\mathrm{d}$ (et)to Monaco li rilascia a sua istanza ed d(et)to Principe co(n) più un Crocifisso piccolino d'argento e fattura di uno specchio regalato al d(et)to Carlo [...]. In piedi del d(et) to Antonio Monaco».

39) ASBNa, Banco dello Spirito Santo, giornale di cassa, 3 aprile 1665, m. 485, p. 374:

"A Carlo d'Angelis vescovo dell'Aquila d(ucati) 55 e p(er) lui ad Andrea Naclerio orefice a comp(imen)to di d(ucati) I94, atteso gli altri d(ucati) I 39.3 l'ha ric(evu) ti con(tan)ti e sono $\mathrm{p}(\mathrm{er})$ tanti spesi in suo nome ad un baculo pastorale d'argento, et a due baldacchini di palmi I 2 e ro guarniti con francia et inforattuno di velluto cremosino napolitano e l'altro d'armesi cremosino».

40) ASBNa, Banco dello Spirito Santo, giornale di cassa, I6 marzo I686, m. 658, p. I 50 (299):

«A Gio(vanni) Matt(e)o Paravicino d(ucati) 82 e $\mathrm{g}(\mathrm{rana}) 2$, et p(er) lui a Dom(eni)co Ant(oni)o Fera a compimento di d(ucati) i 52 e g(rana) is p(er) prez(z)o e fattura di due profumiere d'argento et una mezza corona d'arg(en)to p(er) servi(ti)o di mons(igno)r Paravicino vescovo di Mileto».

4I) ASBNa, Banco dello Spirito Santo, giornale di cassa, 23 giugno I67I, m. 533, p. I068:

«Alli gov(ernato)ri dell'ospit(i)o di Santi Pietro, et Gen(na)ro d(ucati) 70 et p(er) lui a Dom(eni)co Gigante, dis(s)e sono a conto di d(ucati) Ioo, che se li danno, cioè $\mathrm{d}$ (ucati) 64.4.4 per lo prezzo di lib(re) 6 onz(e) 3 d'arg(en)to a compimento di lib(r)e i i onz(e) 2 d'arg(en) to che ha pesato la croce, et pallone d'arg(en)to p(er) esso fatto per sevi(zi)o del confalone dell'loro ospitio, liquidato in $\arg ($ en)to a $\operatorname{rag}($ ion)e de d(uca)ti Io.I.Io la libra, att(e)se l'altre lib(re) 4.I I, percomplim(en)to di $\mathrm{d}(\mathrm{ett}) \mathrm{e}$ lib(re) I I.2 si sono ritenuto dal loro ospit(i)o, per averli consig(na)ti una lampada d'arg(en)to di d(ett)o peso, et li restanti d(ucati) 35. Isono per la manifattura di $\mathrm{d}(\mathrm{ett}) \mathrm{a}$ croce co(n) pallone».

42) ASBNa, Sacro Monte e Banco dei Poveri, giornale di cassa, I9 febbraio 1657 , m. 334, p. n. n.:

«Al cons(iglie)ro pres(iden)te Gio(vanni) Batt(ist)a Amendola d(ucati) I39.2.10 e p(er) lui a Gius(epp)e d'And(re)a d(iss)e p(er) prezzo, et manifattura di due villani et un Orfeo con diversi animalucci d'argento et resta intieram(en)te sodisfatto. In piè con sua firma».

43) ASBNa, Sacro Monte e Banco della Pietà, giornale di cassa, Io ottobre 1665 , m. 562, p. n. n.: «Al p(rincip)e di Cassano d(ucati) so e p(er) lui a Gen(na)ro Monte in conto della fattura di una fontana d'arg(en)to fattali e p(er) lui a Paola [...] p(er) al(trettan)ti».

44) ASBNa, Sacro Monte e Banco della Pietà, giornale di cassa, 5 marzo I665, m. 556 , p. n. n.:

«A Dom(eni)co Vecchio d(ucati) iso et p(er) lui a Dom(eni)co Fera disse a conto di d(ucati) 277 prezzo di un Presepio d'argento di peso (libbra) I77 et li restanti $\mathrm{d}$ (ucati) Ioo sono convenuti di fattura e d(ett)to Presepio ultimato et consi(gna)to al marchese di Altavilla et $\mathrm{p}(\mathrm{er})$ lui a Jacopo Ripetti $\mathrm{p}(\mathrm{er})$ altritanti».

45) ASBNa, Sacro Monte e Banco della Pietà, giornale di cassa, I 2 settembre I665, m. 563, p. n. n.: «A Carlo Mormile d(ucati) 215.4 .5 e p(er) lui a Dom(eni)co Fera disse per prezzo di due statue d'argento a lui consignate, e co(n) q(uest)o pagam(en)to resta intieram(en)te sodisfatto del $\mathrm{d}(\mathrm{ett})$ o prezzo delle due statue, e firma del $\mathrm{d}($ ett)o $\operatorname{Dom}($ eni)co».

46) ASBNa, Banco di San Giacomo, giornale di cassa, i6 maggio I68I, m. 42 I, p. 266:

«Al duca di Diana d(ucati) 296.3.17 e p(er) esso a Dom(eni)co Ant(onio) Fera, e sono cioè d(ucati) 196.3.I7 p(er) il prezzo di lib(br)e 19.I.I/4 di argento e d(ucati) I00 p(er) la manifattura di due carra trionfali d'argento uno fig(uran)te il mare, e l'altro la terra ad esso vend(ut)o e consig(na)to con le loro cassette, e con $\mathrm{d}$ (etto) pag(amen)to resta sodisfatto».

47) ASBNa, Banco di Santa Maria del Popolo, giornale di cassa, 16 maggio I682, m. 509, p. n. n.: «A d(on) Ignazio Provenzale d(ucat)i 33.4.10 e p(e)r esso a Dom(eni) co Antonio Fera et sono per prezzo di due statue d'argento una di Atlante et l'altra d'Ercole fatte di suo ordine a lui contanti con notafede».

48) ASBNa, Banco dello Spirito Santo, giornale di cassa, 24 marzo I683, m. 63I, p. 425 :

«A Fran(ces)co Ant(oni)o Avallone d(ucati) 200 et $\mathrm{p}$ (er) lui a Dom(eni)co Ant(oni)o Fera et sono in conto did(ucati) 527.3.10 quali sono $\mathrm{p}$ (er) il prezzo di due carri et due fontane d'argento di peso di lib(br)e 37.2.3/4 da esso comprate a rag(io)ne di d(ucati) IO.I.Io p(er) libra $\mathrm{d}$ (ucati) $40 \mathrm{p}$ (er) manifattura di dette et $\mathrm{p}$ (er) esso a Nicola Fera p(er) alt(rettant)i».

49) ASBNa, Banco del Popolo, giornale di cassa, 29 maggio I674, m. 298, p. 396:

«A Costantino d'Abisio d(ucati) 44 e p(er) esso a Simone Parascandolo orefice argentiero disse sono p(er) l'intiero prezzo d'una acquasanta a statuette di S(anta) Anna co(n) N(ost)ra S(igno)ra in braccio, San Gios(epp)e e S(an) Gioacchino co(n) aquila tutta d'argento di peso libbre 2 onze ro e una quarta che in denari viene $\mathrm{d}$ (ucati) 29 e p(er) la fattura d'essa d(ucati) I 5 ; in tu(tt)o sono li $\mathrm{d}$ (ett)i d(ucati) 44 a lui venduta e consegnata restando obligato d(ett)o S(igno)r Simone lo d(ett)o argento essere di $\mathrm{t}(\mathrm{ut}) \mathrm{ta}$ perfet(tio)ne e co(n) $\mathrm{d}$ (ett)o pagam(en)to 
resta intieram(en)te sodisfatto, né resta a conseg(ui)re altro, e p(er) esso a Marco Persino p(er) al(trettan)ti, e $\mathrm{p}$ (er) esso ad An(toni)o Baglia p(er) al(trettan)ti».

50) ASBNa, Banco di San Giacomo, giornale di cassa, 28 luglio $\mathrm{I} 674$, m. 374, p. 792:

«Ad Andrea de Ponte d(ucati) so e p(er) esso ad Ant(oni)o Monaco orefice e sono in conto d'una statua d'argento della Madon(n)a che doverà fare consegnarli frà il term(in)e di du mesi dalli 20 di lugl(io) corr(en)te quale statua doverà essere di palmi 3 alta netta di base, e larga a proport(io)ne co(n) essere tutta di getto e d'ogni perfett(ion)e gettata e cicillata e lavorata di sua mano sono convenuti oltre il peso dell'argento darli d(ucati) 70 $\mathrm{p}$ (er) la manifattura lavorat(ion)e et ogn'altra cosa con $\mathrm{f}($ irm) a in piè di del $\mathrm{d}$ (et)to Antonio Monaco».

5 I) ASBNa, Sacro Monte e Banco della Pietà, giornale di cassa, 4 aprile $\mathrm{i} 68 \mathrm{I}, \mathrm{m} .776$, p. n. n.:

«Al marchese de Ugenta d(ucati) 48, e p(er) lui a $\mathrm{m}$ (ast)ro Ottavio de Martino argentiero, $\mathrm{d}($ iss $) \mathrm{e}$ a comp(imen)to de d(ucati) 66, atteso li d(ucati) i 8 l'ha ric(evu)ti con(tan)ti, quali d(ucati) 66 sono $\mathrm{p}(\mathrm{er})$ intiero prezzo, e final pag(amen)to di una figura d'Angelo Custode parte d'argento, e parte de rame indorata, con sua pedagna d'ebano et tartuca similm(en)te guarnita d'arg(en)to, et rame indorata, et da esso venduta, et consig(na)ta cossì d'accordo tra loro, et co(n) detto pag(amen)to resta intieram(en)te sodisf(at)to, co(n) fir(m)a del d(ett)o Ottavio de Martino».

52) ASBNa, Sacro Monte e Banco della Pietà, giornale di cassa, 4 aprile $\mathrm{i} 68 \mathrm{I}, \mathrm{m} .775$, p. n. n.:

«Al duca Valentino d(ucati) 4I.I.I et $\mathrm{p}$ (er) lui a Gio(vanni) Dom(eni)co Vinaccia sono ciò̀ d(ucati) 38.r. I p(er) libbre et concio 7 d'argento in guarnizioni di scopette, candelieri, un S(an) Sebastiano, et uno scaravattiello, et $\mathrm{d}$ (ucati) $3 \mathrm{p}$ (er) manifatt(u)ra $\mathrm{p}(\mathrm{er})$ tanti consig(na)ti et fatti $\mathrm{p}(\mathrm{er}) \operatorname{serv}(\mathrm{iti}) \mathrm{o}$ di casa sua et con $\mathrm{d}$ (ett)o pag(amen)to resta da lui intieramente sodisfatto $\mathrm{p}$ (er) tutto lo $\mathrm{p}$ (rossi)mo del corr(en)te, et non resta a conseg(ui)re cosa alcuna et $\mathrm{p}$ (er) lui a Paulo Salombrino $\mathrm{p}(\mathrm{er}) \mathrm{al}(\operatorname{trettant}) \mathrm{i}$.

53) ASBNa, Sacro Monte e Banco della Pietà, giornale di cassa, I 6 aprile I 68 I, m. 777, p. n. n.:

«A d(on) Dom(eni)co del Giudice d(ucati) 35.4. I0 e p(er) lui a Dom(eni)co Regi orefice argentiero, e cono a compim(en)to de d(ucati) 40, atteso l'altri l'ha rice(vu)ti de con(tan)ti e sono $\mathrm{p}(\mathrm{er})$ uno paro de piramidette d'argento con due statuette una del glorioso San Gaetano, et l'altra del glorioso San Nicolò di peso lib(bre) 3, e un'onza, e mola d'arg(en)to; et [...] p(er) la fattura d'esse con una bacchetta d'argento e con questa resta sodisfatto et $\mathrm{p}(\mathrm{er})$ lui di esso medesimo».

54) ASBNa, Sacro Monte e Banco della Pietà, giornale di cassa, 24 maggio I68 I, m. 775, p. n. n.: «Ad Onofrio di Castro d(ucati) 33 et p(er) lui a Nicola d'Aula e sono $\mathrm{p}$ (er) saldo et final pag(amen)to di tutti li lavori d'arg(en) to et oro, et di ogni altro fatto $\mathrm{p}$ (er) serv(iti)o del principe della Torella restando con questo pagamento sald(at) o [...]. Con firma di d(ett)o Nicola d'Aula».

55) ASBNa, Banco di San Giacomo, giornale di cassa, 28 maggio I665, m. 298, p. 528:

«A d(on) Cristofano Ruiz de Pedrosa d(ucati) roo e $\mathrm{p}($ er) esso a Jacinto Portio argentiero dell'em(inenti)ssimo sig(no)r cardinale d'Aragona e sono in co(n)to d'un Cristo d'argento che fa d'ord(in)e di s(ua) em(inenz)a e $\mathrm{p}($ er) esso ad Aniello Treglia p(er) alt(rettan)ti, e p(er) esso a Matteo Grimaldo p(er) alt(rettan)ti».

56) ASBNa, Sacro Monte e Banco della Pietà, giornale di cassa, Io aprile I668, m. 59I, p. n. n.:

«A d(on) Carlo Pignatello d(ucati) is, e p(er) lui a Gennaro Monte argentiero a comp(imen)to di d(ucati) 25, stante gli altri d(ucati) ro l'ha rice(vuti) da esso con(tanti) e d(ucati) 25 sono p(er) lo peso e fattura d'uno reliquiario d'arge(n)to da lui fatto, et ad esso conseg(na) to e $p($ er) lui a Geronimo Simone $p(e r)$ alt(rettant)i, e $\mathrm{p}(\mathrm{er})$ lui a Jac(op)o Ripetto p(er) alt(rettant)i».

57) ASBNa, Banco di Sant'Eligio, giornale di cassa, I I aprile $\mathrm{I} 674, \mathrm{~m} .389$, p. n. n.:

«A Gius(epp)e Califano d(ucati) ss e p(er) lui al p(ad) re fra Innocentio d'Arsano p(er) altr(ettant)i, e p(er) lui ad Ignazio Strozza a comp(imen)to di ducati 223 atteso l'altri l'ha ricevuti parte in contanti e d(ucati) I $30 \mathrm{p}$ (er) il banco di S(an) Jac(op)o in testa di Dom(eni)co Strozza e dal d(ett)o girata a d(on) Carlo Ferra, e dal d(ett)o girata al d(ett)o rev(eren)do p(ad)re maestro Innocentio Arzano $\mathrm{p}$ (er) causa d'una statuetta d'argento della Beatiss(im)a Vergine che $\mathrm{d}($ ett)o Ignazio s'obligò fare come $\mathrm{p}(\mathrm{er})$ albarano a 25 nov(embr)e 1673 p(er) mano di n(ota)re Pietro Piscopo come si contiene anco in $\mathrm{d}(\mathrm{ett}) \mathrm{a}$ fede di $\mathrm{d}$ (ucati) I 30 di S(anto) Giac(om)o, et essendo stata fatta $\mathrm{d}$ (ett)a statuetta e consignata a sua sodisfatione si è stracciato $\mathrm{d}($ ett)o albarano e con il pre(sen)te pagam(en)to s'intenda anco rotta e chiusa $\mathrm{d}$ (ett)a partita di banco di S(an) Giac(om)o, ne d(ett)o Ignatio restà a conseguire cos'alc(u) na da lui $\mathrm{p}($ er $) \mathrm{d}($ ett)o effetto, e lui sodisfatto $\mathrm{p}(\mathrm{er}) \mathrm{d}(\mathrm{ett}) \mathrm{a}$ consegna dichiarando d'essere stata di tutta sua sodisfatione, e p(er) lui a Fran(ces)co Califano per altrit(ant)i».

5) ASBNa, Banco dello Spirito Santo, giornale di cassa, 9 maggio I68 I, m. 6is, p. 678:

«A d(on) Gio(vanni) Ant(oni)o Olivieri d(ucati) 42 et $\mathrm{p}(\mathrm{er})$ lui a Biagio Coraggio; disse sono $\mathrm{p}(\mathrm{er})$ intiero prezzo d'un quadro d'arg(en)to con cornice d'ebano guarnita parim(en)ti guarnita d'argento ad esso venduto; et conseg(na)to di tutta sua sodisfatt(io)ne et p(er) esso à Gios(epp)e Belli p(er) altrettanti».

59) ASBNa, Banco di San Giacomo, giornale di cassa, 22 dicembre I68I, m. 425 , p. 785 :

«A Gio(vanni) Bat(tis)ta Tocco d(ucati) 200 e p(er) esso ad And(re)a Finelli e sono p(er) il prezzo di sei sta- 
tuette d'argento con fiori naturali ad esso venduti che li doverà consig(na)re $\mathrm{p}(\mathrm{er})$ tutto li io corr(en)te e $\mathrm{p}(\mathrm{er})$ esso ad Capuano $\mathrm{p}(\mathrm{er})$ alt(rettant)i».

6o) ASBNa, Banco dello Spirito Santo, giornale di cassa, 5 ottobre I682, m. 628, p. 233 :
«A Nicolò Fiorentino d(ucati) 62.2 et p(er) lui a Gios(epp)e Maiorino disse sono p(er) lo prezzo di due statue d'argento di peso 5.53 L[libbra ?] ad esso venduto et consignato $\mathrm{p}$ (er) detto prezzo così d'accordo et con questo pagam(en)to resto' intieramente sodis(fat)to del prezzo d'esse ne $\mathrm{p}(\mathrm{er})$ altra quals(iasi)a causa se li resto cos'alcuno». 
1. Dedico questo saggio alla memoria di Elio Catello (1927-2017). Vorrei, inoltre, ringraziare Giovanni Boraccesi, Simona Carotenuto, Alessandro Giunnelli, Sabrina Iorio, Ida Mauro, Giuseppina Merola e Andrea Zappulli.

2. C. Catello (1984), «Argexnti», in Civiltà del Seicento a Napoli, Museo di Capodimonte - Museo Pignatelli, Napoli, vol. II, p. 307308; E. Catello e C. Catello (2000), Scultura in argento nel Sei e Settecento a Napoli, Napoli, p. 27-57; R. Ruotolo (2009), Per un profilo della storiografia delle arti decorative a Napoli in eta barocca, in Ritorno al Barocco: da Caravaggio a Vanvitelli, diverse sedi, Napoli, vol. II, p. 108-115.

3. Sull'argomento si veda in ultimo: Il tesoro di Napoli. I capolavori del Museo di San Gennaro, Fondazione Roma Museo Palazzo Sciarra, Roma, 2013.

4. C. DE Lellis (1689 circa), Aggiunta alla "Napoli sacra" dell'Engenio Caracciolo, Napoli, vol. II, p. 272 (edizione informatizzata a cura di E. Scirocco; M. Tarallo; S. De Mieri, p. 190, <www. memofonte.it>).

5. Appendice documentaria 1.

6. E. Nappi (2017-2018), «Il Sant'Antonio da Padova di Cerreto Sannita e nuove acquisizioni sugli argentieri Giovanni Maiorino e Aniello Treglia», in Ricerche sull'arte a Napoli in età moderna, p. 138.

7. Un trentennio prima il religioso incaricava, in data 5 settembre 1637, Andrea Finelli di eseguire due statue d'argento da destinarsi, verosimilmente, alla sagrestia di San Domenico Maggiore di Napoli, come si evince dalla polizza in E. NAPPI (2015), «La chiesa e il convento di San Domenico Maggiore di Napoli: documenti», Ricerche sull'arte a Napoli in età moderna, p. 48.

8. Appendice documentaria 2.

9. E. Nappi, «La chiesa e il convento...», op. cit., p. 48 .

10. C. Celano (1692), Notitie del bello, dell'antico e del curioso della città di Napoli [...], Napoli, vol. vII, p. 7.

11. C. Celano, Notitie del bello..., op. cit., vol. II, p. 136-137.

12. Sull'artista si rimanda, in ultimo, a A. Russo (2016), Il Barocco "peregrino" di Giovan Domenico
Vinaccia. Il paliotto argenteo dell'altare maggiore, San Gennaro patrono delle arti. Conversazioni in cappella 2016, a cura di S. Causa, Napoli, p. 37-77. Per una scansione degli interventi di Vinaccia, sebbene tutti da aggiornare, si rimanda alla scheda di G. GuIdA, in Civiltà del Seicento..., op. cit., vol. II, p. 233-237.

13. B. De Dominici (1742-1743), Vite de' pittori, scultori ed architetti napoletani, Napoli, vol. III, p. 164.

14. Appendice documentaria 3-4.

15. Appendice documentaria 5.

16. Appendice documentaria 6. Su Monte si veda A. Catello, in Civiltà del Seicento..., op. cit., vol. II, p. 305.

17. E. Catello (1979), «Gian Domenico Vinaccia e il paliotto di San Gennaro", Napoli Nobilissima, 18, p. 73-144; V. Rizzo (1984), «Documenti su Cavallino, Corenzio, De Matteis, Giordano, Lanfranco, Solimena, Stanzione, Zampieri ed altri, dal 1636 al 1715", in Seicento napoletano. Arte, costume e ambiente, a cura di R. Pane, Milano, p. 392.

18. G. Filangieri (1883-1891), Documenti per la storia le arti e le industrie delle provincie napoletane raccolti e pubblicati per cura di Gaetano Filangieri Principe di Satriano, Napoli, vol. vi, p. 193; F. Strazzullo (1978), La Real Cappella del Tesoro di S. Gennaro. Do cumenti inediti, Napoli, p. 96-97; V. Rizzo, «Documenti su Cavallino...», op. cit., p. 392; G. GUIDA (2009), "Alcuni artisti dei secoli XVII-XIX nelle carte dell'Archivio Storico del Banco di Napoli-Fondazione», Quaderni dell'Archivio Storico Fondazione Banco di Napoli 2007-2008, p. 384

19. G. Artale (1672), Alloro fruttuoso, Napoli, p. 116.

20. Appendice documentaria 7.

21. E. Catello e C. Catello (1973), Argenti napoletani dal XVI al XIX secolo, Napoli, p. 96.

22. Da associare a quella «bellissima statua anco d'argento, fatta dal Monti con il braccio di San Biagio» osservata da Celano e Parrino in detta chiesa. Cfr. C. Celano, Notitie del bello..., op. cit., vol. II, p. 216; D. A. Parrino (1700), Napoli città nobilissima, antica e fedelissima, esposta agli occhi et alla mente de' curiosi, Napoli, I, p. 198

23. E. NAPpi (2010-2011), «Il complesso domenicano dei Santi
Pietro e Sebastiano di Napoli. Nuove notizie», in Ricerche sul '600 napoletano, p. 144.

24. Appendice documentaria 8.

25. Appendice documentaria 9.

26. Difficile associarla a quella ivi conservata. Cfr. E. Catello e C. Catello, Scultura in argento nel Sei e Settecento a Napoli..., op. cit., p. 256

27. Appendice documentaria 10.

28. Il nome del beato si evince in modo inequivocabile dalla bancale del 16 febbraio 1674. Cfr. ASBNa, Sacro Monte e Banco dei Poveri, bancale, m. 2721.

29. Appendice documentaria 11.

30. A. Parrino, Napoli città nobilissima..., op. cit., vol. I, p. 194; G. Sigismondo (1788-1789), Descrizione della città di Napoli e suoi borghi, Napoli, vol. II, p. 25.

31. E. NAPPI, «La chiesa e il convento...», op. cit., p. 48.

32. E. Catello (1998-1999), «Argenti napoletani del Seicento: considerazioni su documenti inediti», in Ricerche sul '600 napoletano, p. 14.

33. Identificabile con quella «magnifica e ben lavorata statua d'argento» vista da Celano nella sagrestia di San Pietro a Maiella. Cfr. C. Celano, Notitie del bello..., op. cit., vol. II, p. 208.

34. Appendice documentaria 12.

35. V. DEL VAsto (1995), Baroni nel tempo. I Tocco di Montemiletto dal XVI al XVIII secolo, Napoli, p. 130-132.

36. Appendice documentaria 13.

37. Appendice documentaria 14.

38. B. De Dominici, Vite de' pittori..., op. cit., vol. III, p. 164.

39. V. Rizzo, «Documenti su Cavallino...», op. cit., p. 407; V. Rizzo (2001), Lorenzo e Domenico Antonio Vaccaro. Apoteosi di un binomio, Napoli, p. 217.

40. E. Catello, Argenti napoletani del Seicento..., op. cit., p. 13; E. NAPPI (2002-2003), «I Gesuiti Napoli. Nuovi documenti», in $R i$ cerche sul' 600 napoletano, p. 129.

41. Appendice documentaria 15. Sull'attività di Paolo Carafa nel duomo di Aversa si veda A. GRIMALDI (2010), La decorazione del 
duomo di Aversa in età moderna. Storia di una committenza tra aristocrazia e clero, Napoli, p. $42-43,120$.

42. G. G. Borrelli (2008), Il Museo Diocesano di Napoli. Percorsi difede e arte, a cura di P. LEONE De Castris, Napoli, p. 210-211, cat. 93.

43. Il busto è pubblicato come di ignoto argentiere napoletano e collegato correttamente alla committenza di Paolo Carafa in E. Catello e C. Catello, Scultura in argento nel Sei e Settecento a Napoli..., op. cit., p. 71, cat. Iv.

44. Cfr. A. Catello, in Civiltà del Seicento..., op. cit., vol. II, p. 321324, cat. 5.13, 5.17; E. CaTEllo e C. Catello, Scultura in argento nel Sei e Settecento a Napoli..., op. cit., p. 81, cat. XVIII, 89, cat. XxviII.

45. Appendice documentaria 16.

46. V. Rizzo (1984), «Scultori della seconda metà del Seicento", in Seicento napoletano. Arte, costume $e$ ambiente, a cura di R. PANE, Milano, p. 295, doc. 2; G. FORGIONE (2013-2014), Il complesso dei Girolamini: Artisti e committenti nella Napoli del Seicento, tesi di dottorato, Università degli Studi di Napoli «Federico II», XxvII ciclo, p. 118-120.

47. P. SARnelli (1685), Guida de' forestieri curiosi di vedere e d'intendere le cose più notabili della regal città di Napoli e del suo amenissimo distretto, Napoli, vol. I, p. 118-119; C. Celano, Notitie del bello..., op. cit., vol. II, p. 103.

48. C. Celano, Notitie del bello..., op. cit., vol. III, p. 318.

49. Appendice documentaria 24.

50. Appendice documentaria 25.

51. In quest'area non è noto alcun esemplare di Vinaccia. Cfr. A. RicCO (2017), Manufatti in argento tra '500 e '600 nel basso Salernitano tra modelli toscani e botteghe napoletane: le croci astili dalla simbologia Arbor vitae, in Ritorno al Cilento. Saggi di storia dell'arte, a cura di F. Аввате e A. Ricco, Foggia, p. 67-74.

52. Appendice documentaria 26.

53. A. Pinto (2018), Raccolta notizie per la storia, arte, architettura di Napoli e contorni. Parte 1: artisti e artigiani, p. 3379.

54. Appendice documentaria 27.
55. C. Borromeo (1577), Instructiones fabricae et supellectilis ecclesiasticae, Roma, p. 22.

56. Cfr. R. C. Leardi (2012), «Riflessioni sul ciborio nella Napoli del Seicento fra tradizione e innovazione. Una custodia "ritrovata" di Francesco Balsimelli florentinus», in M. Panarello, Fanzago e fanzagbiani. Il circuito artistico nel Seicento tra Roma, Napoli e la Sicilia, Soveria Mannelli, p. 505-527.

57. C. Celano, Notitie del bello..., op. cit., vol. vi, p. 38-39.

58. Per lo stesso luogo Vinaccia consegnò nel 1682 ben sei paliotti. Cfr. E. Catello, Gian Domenico Vinaccia..., op. cit., p. 123.

59. Appendice documentaria 28.

60. Appendice documentaria 29.

61. E. Catello e C. Catello, $A r$ genti napoletani dal XVI..., op. cit., p. 142-143.

62. Appendice documentaria 30.

63. Appendice documentaria 31. Cfr. anche ASBNa, Banco di San Giacomo, giornale di cassa, 13 luglio 1674, m. 373, p. 779.

64. Appendice documentaria 32.

65. Appendice documentaria 33.

66. Vita della venerabile madre D. Maria Carafa sorella di Paolo $I V$ papa e fondatrice del monistero di S. Maria della Sapienza, coll'aggiunta delle vite della ven. suor Francesca del Ss. Sacramento e di S. Caterina da Genova, ristrette da un sacerdote napolitano [...], $\mathrm{Na}$ poli, 1773, p. 497, n. XCVII.

67. C. Celano, Notitie del bello..., op. cit., vol. II, p. 36 .

68. Appendice documentaria 34.

69. C. Celano, Notitie del bello..., op. cit., vol. Iv, p. 9.

70. Appendice documentaria 3536.

71. C. Celano, Notitie del bello..., op. cit., vol. Iv, p. 7.

72. P. SARNELLI, Guida de' forestieri..., op. cit., vol. II, p. 259.

73. Appendice documentaria 37.

74. E. Catello e C. Catello, Scultura in argento nel Sei e Settecento a Napoli..., op. cit., p. 75-77, cat. X-XI.
75. C. Celano, Notitie del bello..., op. cit., IV, p. 100.

76. Appendice documentaria 38.

77. Appendice documentaria 39.

78. Appendice documentaria 40.

79. Appendice documentaria 41.

80. C. Celano, Notitie del bello..., op. cit., vol. II, p. 32 .

81. Appendice documentaria 17.

82. Appendice documentaria 18.

83. Appendice documentaria 19.

84. Sulla presenza di paliotti di fattura napoletana in Puglia si rimanda a G. BoracCESI (2015), «Argenti napoletani di età barocca nella Cattedrale di Brindisi», Parola e storia, 17, p. 96, nota 39 .

85. Appendice documentaria 20.

86. Cfr. il contratto notarile: Archivio di Stato di Napoli, Notai del xvir secolo, Domenico Cardamomo, 1221/4, cc. 150-151.

87. Sull'argentiere si rimanda a A. Catello, in Civiltà del Seicento..., op. cit., vol. II, p. 305-306.

88. Appendice documentaria 21.

89. Appendice documentaria 22.

90. Appendice documentaria 23.

91. P. SARnElli, Guida de' forestieri..., op. cit., vol. II, p. 303.

92. E. Catello e C. Catello, Scultura in argento nel Sei e Settecento a Napoli..., op. cit., p. 235, doc. v.

93. V. Rizzo (1987), «Altre notizie su pittori, scultori ed architetti napoletani del Seicento (dai documenti dell'Archivio Storico del Banco di Napoli)», in Ricerche sul ‘600 napoletano, p. 162.

94. Appendice documentaria 42.

95. Appendice documentaria 43.

96. E. Catello, Argenti napoletani del Seicento..., op. cit., p. 12; F. Strazzullo (1986), «Alcuni documenti inediti attinenti la storia dell'arte del '600 napoletano", in $R i$ cerche sul '600 napoletano, 5, p. 244.

97. Appendice documentaria 44.

98. Appendice documentaria 45.

99. Appendice documentaria 46. 
100. G. Labrot (1992), Collections of Paintings in Naples 1600-1780, Monaco, p. 176

101. Appendice documentaria 47.

102. R. C. LEARDI (2017), «Il ciclo ritrovato di Bernardino Fera nella 'sacra grotta' di Santa Maria della Sanità», in La chiesa e le catacombe di San Gaudioso. Storia di un restauro, Napoli, p. 45.

103. Appendice documentaria 48.

104. Sull'orefice si veda C. CatelLO, Argenti, in Civiltà del Seicento..., op. cit., vol. II, p. 307.

105. Appendice documentaria 49.

106. Appendice documentaria 50.

107. G. B. D’Addosio (1913), «Documenti inediti di artisti na- poletani del vi e del xvir secolo", Archivio Storico per le Provincie Napoletane, 38, p. 46; F. STRAZZULLO (1954), «La vertenza tra Cosimo Fanzago e la deputazione del Tesoro di S. Gennaro", Archivio storico per le province napoletane, 34, p. 79; A. Delfino (1985), «Documenti inediti per alcuni pittori napoletani del '600 e l'inventario dei beni lasciati da Lanfranco Massa, con una sua breve biografia (tratti dall'Archivio Storico del Banco di Napoli e dall'Archivio di Stato di Napoli)», in Ricerche sul '600 napoletano, 5, p. 99.

108. Appendice documentaria 51.

109. Appendice documentaria 52.

110. Appendice documentaria 53.

111. Appendice documentaria 54.
112. Appendice documentaria 55.

113. E. Catello e C. Catello, Argenti napoletani dal XVI..., op. cit., p. 61.

114. Appendice documentaria 56.

115. Appendice documentaria 57.

116. Appendice documentaria 58.

117. A Napoli, a detta di Celano, si lavoravano «fiori d'argento così al naturale, che loro non manca altro che l'odore ed il colore; ed io confesso simili non haverne veduti in Italia». C. Celano, Notitie del bello..., op. cit., vol. I, p. 25.

118. Appendice documentaria 59.

119. Appendice documentaria 60. 